\title{
Hybrid Propulsion Parametric and Modular Model: a novel engine analysis tool conceived for design optimization
}

\author{
A. Mogavero* I. Taylor ${ }^{\dagger}$ R. E. Brown ${ }^{\ddagger}$ \\ Centre for Future Air-Space Transportation Technology, \\ University of Strathclyde, Glasgow, G1 1XJ, United Kingdom
}

\begin{abstract}
The development of a new, efficient means of access to space would yield a giant leap in the viability of many proposals for space exploitation and exploration that are currently being considered. One proposal is to employ so-called 'space planes,' designed to follow an airliner-like mission profile, in order to achieve the considerable savings in the cost of access to space that will be required. Further development of propulsion technology, particularly air-breathing propulsion systems, is fundamental to yielding a practical space plane, however. Although the development of such technology for high speeds is still in its infancy, in recent years some promising full scale tests of the so-called scramjet engine concept have been performed. This paper describes initial steps towards the construction of an engineering tool, called the Hybrid Propulsion Parametric-Modular Model, which can be used to study the behaviour of scramjets through the use of simplified numerical models, aided and supported by advanced Computational Fluid Dynamic simulations and experimental data where appropriate. The Hybrid Propulsion Parametric-Modular Model is conceived to be modular and flexible, and to be fully parametric. It is intended to facilitate the consideration, from the earliest phases of the design process, of several critically-important aspects of the propulsion system and its interaction with the vehicle. The greatest benefit of the model, given its limited computational cost, is expected to be however in the performance of multi-disciplinary optimization studies where it will be employed as part of a more generic systems-based model for characterising the performance of the next generation of space access vehicles.
\end{abstract}

\section{Glossary}

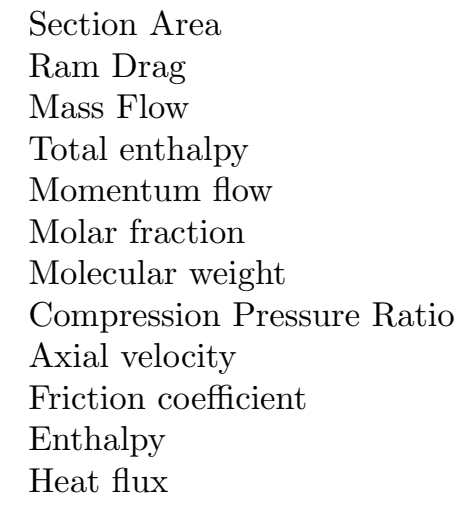

$\dot{m} \quad$ Mass flow rate

$p \quad$ Static pressure

St Stanton number

$\gamma \quad$ ratio of specific heats

$\eta \quad$ Efficiency

$\rho \quad$ Density

$\tau \quad$ Friction stress

$\Phi \quad$ Stoichiometric composition

$\varphi \quad$ Equivalence ratio

$\theta \quad$ Injection angle

*PhD Student, Student Member AIAA.

${ }^{\dagger}$ Lecturer.

${ }^{\ddagger}$ Director and Professor, Senior Member AIAA. 


\section{Subscripts}

$\begin{array}{llll}f & \text { Fuel } & 1 & \text { Beginning of the module } \\ o x & \text { Oxider } & 2 & \text { End of the module } \\ W & \text { Wall conditions } & 3 & \text { Module third node } \\ \infty & \text { Free stream conditions } & & \\ 0 & \text { Stagnation or total conditions } & & \end{array}$

\section{Introduction}

Decades after the first pioneering years of space exploration, there is still a large margin for improvement within the field of space transportation technology. In particular, the development of a new, efficient means of access to space would yield a giant leap in the viability of many proposals for space exploitation and exploration that are currently being considered. One possible strategy for making access to space cheaper, more flexible and reliable is the Single-Stage to Orbit (SSTO) concept. The idea is to supplant current expendable launch vehicle technology by developing a class of re-usable 'space planes' that are able to reach orbit and then re-enter the atmosphere by following a mission profile that is very similar to that used by airliners. The technological challenges of putting this very simple idea into practice are significant, however, given the complexity of such a system in reality. Further development of propulsion technology is fundamental to yielding a practical SSTO system. Indeed, engines with very much higher propulsive efficiency than is currently achievable, particularly at high speed and altitude, will be required to power vehicles that are able to carry an economically viable payload into orbit. Air breathing propulsion systems have the advantage of using the atmosphere as part of their propellant, giving them a major advantage in efficiency over the rocket systems that have been used in all forms of space transportation up to now. Indeed, the development of efficient air-breathing spacecraft propulsion is seen as the key to improving the reliability and flexibility of the SSTO concept and allowing it to become airline-like in practice. Despite the fact that air-breathing propulsion is widely employed for airliners that fly at subsonic and low supersonic speeds, suitable technology for the higher speeds required of SSTO vehicles is still in its infancy. In recent years, however, some full scale tests of the scramjet engine concept have been performed at hypersonic flight speeds, and prospects so far for this technology are very promising. ${ }^{1}$

The research described in this paper aims to understand the behaviour of propulsion systems, through the use of simplified numerical models, using advanced Computational Fluid Dynamic (CFD) computations and experimental data to verify, validate and extend the research where appropriate. The overall goal is to construct a system model of scramjet behaviour which can be used in the design of future SSTO systems. In this vein, this scramjet model is designed to form part of a more comprehensive vehicle system model in order to carry out vehicle system analyses and also Multi-disciplinary Design Optimization (MDO).

Many examples of simplified propulsion system models are described in the published literature; see for instance the review by Tran. ${ }^{2}$ Notwithstanding this, the source code of such models is rarely available, and individual models are often dedicated only to a particular application. A further aim of this research is thus to provide a highly modular model that is able to analyse a wide range of advanced propulsion configurations. Particularly given its intended application in MDO, the software of the model is designed to place a high priority on computational efficiency and speed of execution.

\section{Simplified Model}

We are in the process of developing an engineering-level, modular software package, called the Hybrid Propulsion Parametric-Modular Model (HPPMM), for the design and analysis of advanced aircraft propulsion systems - in particular, advanced hybrid propulsion systems based around the scramjet engine concept. The terminology 'hybrid' is taken to denote any possible combination of propulsion technology, either when installed separately or when integrated into a single engine. Usually the former is called combination propulsion and the latter is called composite propulsion ${ }^{3}$ or, more frequently, combined cycle propulsion. ${ }^{4}$ The Hybrid Propulsion Parametric-Modular Model (HPPMM) software is developed in the Matlab® environment. The software has a modular structure that takes advantage of the intrinsic modularity of the object oriented programming formalism embodied within Matlabß). The modularity brings flexibility to the soft- 
ware allowing it to be configured to easily model many different kinds of propulsion systems. Moreover, thanks to the object oriented structure of the software, it is possible to implement every module in terms of a defined set of properties and parameters that can be changed easily during the run-time. This latter feature makes the software particularly suitable for MDO applications.

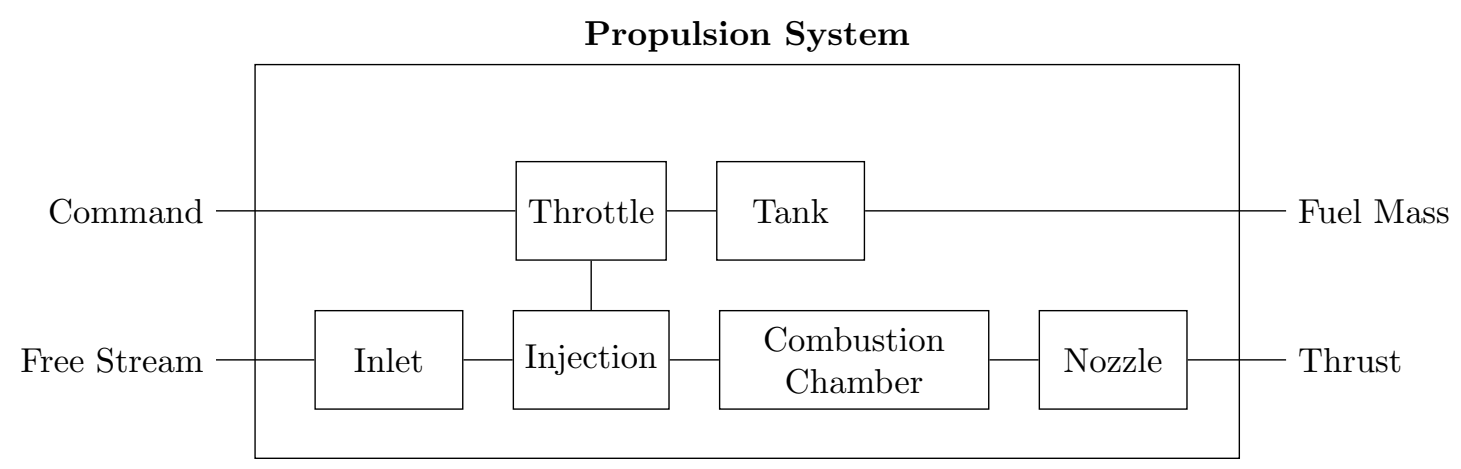

Figure 1. Example of the modular structure of the propulsion model for a Scramjet/Ramjet.

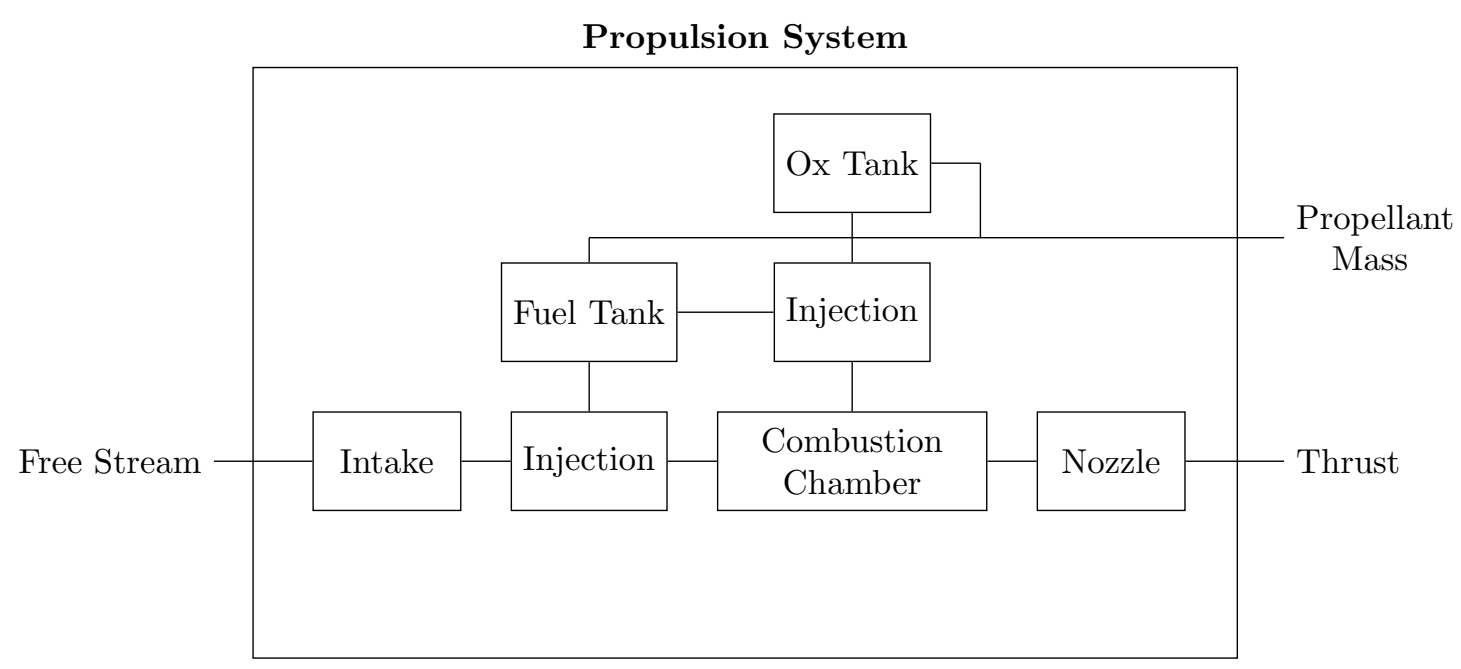

Figure 2. Example of the modular structure of the propulsion model for a RBCC engine.

In Fig. 1, for instance, a schematic of the model, structured in order to represent a scramjet/ramjet engine, is depicted. The modules can easily be changed and re-arranged to represent an alternative engine configuration. Hybrid engines can be modelled by collating and interconnecting all the required modules, then switching on/off certain of the modules according to a proposed schedule of operation. In Fig. 2 for instance, an example of the model configured to represent a Rocket-Based Combined Cycle (RBCC) propulsion system is illustrated.

\section{II.A. Gas dynamics models}

Before describing each individual engine module in detail, it is appropriate to summarise the general gas dynamic models that are used as basis for physical modelling of the flow within all of the engine components. The gas dynamic modules that have so far been implemented are the following:

- isentropic one-dimensional compression/expansion ${ }^{5}$

- Fanno flow ${ }^{5}$

- Rayleigh flow ${ }^{5}$

- One dimensional balance equation solver (see II.A.1) 
These models are embodied as independent modules within the code in order to give flexibility to the software. The first three models consider a perfect gas, in other words one with constant ratio $\gamma$ of specific heats. For present purposes, however, they are also employed when a gas with variable $\gamma$ is modelled, with the proviso thus that the changes in $\gamma$ of the gas within each engine module are small.

\section{II.A.1. Balance equation solver}

While all the other gas dynamic models are well described in the literature, ${ }^{5}$ it is appropriate to give more detail regarding the balance equation solver that is implemented within HPPMM. This part of the model solves the equations

$$
\left\{\begin{aligned}
\rho_{1} U_{1}-\Delta G & =\rho_{2} U_{2} \\
p_{1}+\rho_{1} U_{1}^{2}-\Delta I & =p_{2}+\rho_{2} U_{2}^{2} \\
h_{1}+\frac{U_{1}^{2}}{2}-\Delta H & =h_{2}+\frac{U_{2}^{2}}{2}
\end{aligned}\right.
$$

representing, respectively, the balance of mass, momentum and energy along a duct that has constant section area. In these equations, $\Delta G, \Delta I$ and $\Delta H$ represent respectively the flux of mass, momentum and energy across the walls of the duct. The solver attempts to determine the conditions at state 2 (i.e. at the end of the duct) given the conditions at state 1 (i.e. at the beginning of the duct) and these lateral fluxes. In order to simplify the solution process the system of equations is rewritten in the following form:

$$
\left\{\begin{aligned}
\rho_{1} U_{1}-\Delta G & =\rho_{2} U_{2} \\
p_{1}+\rho_{1} U_{1}^{2}-\Delta I & =\left(1+\gamma M_{2}^{2}\right) p_{2} \\
h_{1}+\frac{U_{1}^{2}}{2}-\Delta H & =h_{2}+\frac{\gamma M_{2}^{2} R T_{2}}{2}
\end{aligned}\right.
$$

it is important to note that the only assumption needed to support the validity of Eq. (2) is that the gas be ideal in its behaviour; the ratio of specific heats $\gamma$ can thus be modelled as a function of temperature. The solution process starts by using a tentative value for $M_{2}$ and then proceeds through the solution of each element of Eq. (2) in turn. First the energy equation is solved in terms of the static temperature $T_{2}$ by means of a bisection algorithm, then the momentum equation is used to determine the static pressure $p_{2}$ and finally the the mass balance equation is used to check whether the tentative value assumed for $M_{2}$ is correct or not. The whole procedure is then iterated by means of a bisection algorithm until the desired level of convergence is reached.

\section{II.B. Intake}

The intake model considers the four stations depicted in Fig. 3. To allow the possibility of modelling a non-adapted intake, the conditions at station $S_{1}$ can be assumed to be different from those at station $S_{i n}$. The external flow path in the case of a non-adapted intake is assumed to be isentropic if the external flow is subsonic, whereas if the external flow is supersonic then a normal shock is assumed to be present and to be sufficiently strong to adapt the intake fully to the external flow. Whenever the intake is not fully adapted, an intake drag 6

$$
D=\dot{m}_{i n}\left(U_{i n}-U_{1}\right)+A_{i n}\left(p_{i n}-p_{1}\right)
$$

is calculated, subsequently to be incorporated within the overall thrust calculation for the engine.

The remainder of the flow within the intake is calculated by considering a total pressure drop across the convergent part of the duct and assuming the flow in the divergent part of the duct to be isentropic (see II.A). The pressure drop is given by MIL Spec E-5007D ${ }^{4}$ in the case of subsonic conditions at station $S_{i n}$ and by fitting to a prescribed curve ${ }^{7}$ otherwise (see Fig. 4).

\section{II.C. Fan and Compressors}

A simple model for fans and compressors is also provided. The model takes as input the total pressure ratio $R_{p}$ and considers the compression to be isentropic. The stagnation conditions at the end of the compressor are thus

$$
p_{02}=R_{p} p_{01}, \quad \rho_{02}=R_{p}^{\frac{1}{\gamma}} \rho_{01}, \quad T_{02}=\frac{1}{R} \frac{p_{02}}{\rho_{02}}
$$




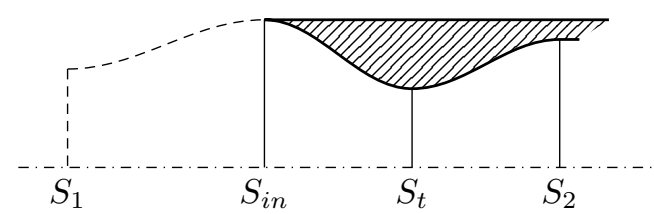

Figure 3. Definition of stations for the intake model.

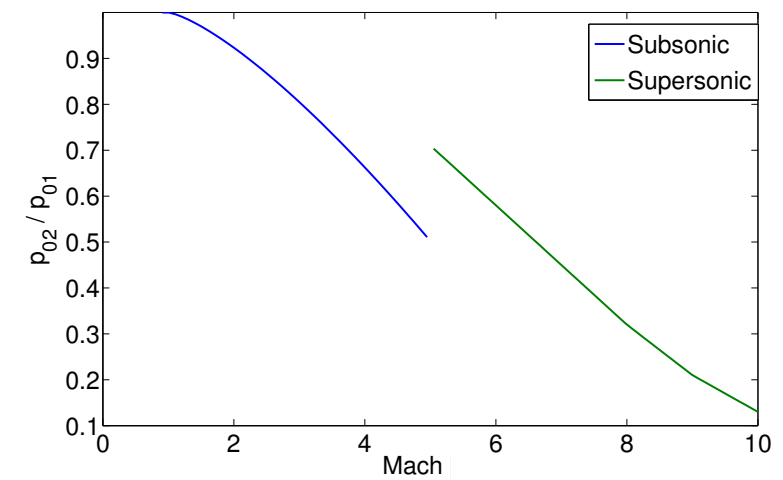

Figure 4. Intake total pressure drop as a function of Mach number. ${ }^{7}$

The static conditions and the Mach number at the end of the compressor are then calculated by imposing a mass balance across the compressor. This balance equation is solved iteratively using the Mach number as the tentative variable.

\section{II.D. Mixer}

The mixer module allows the mixing of two flows to be modelled. It is therefore defined as a module with an additional internal node (node 3) which can be joined to any other type of module within the HPPMM toolbox. This module is typically used when the momentum contribution of the flow out of the module attached to the internal node cannot be neglected. A typical example of where the mixer module would be used is in a model of an ejector system, where a rocket plume mixes with the airflow coming from the inlet (see for instance IV.B and IV.C).

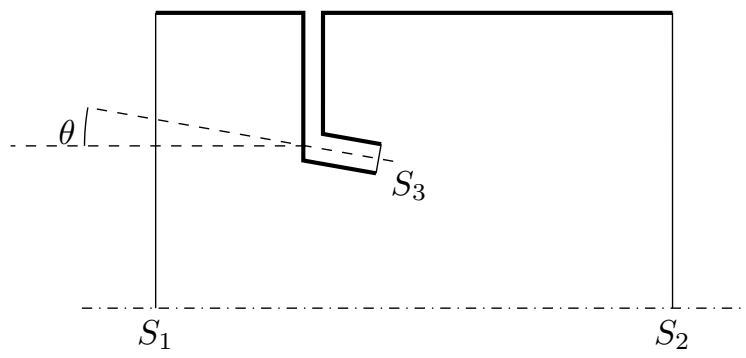

Figure 5. Definition of stations for the mixer module.

This model uses the solver described in II.A.1; the fluxes needed in Eq. (1) are

$$
\left\{\begin{aligned}
\Delta G & =-\frac{A_{3}}{A_{1}} \rho_{3} U_{3} \\
\Delta I & =\eta\left[\Delta G U_{3}-\frac{A_{3}}{A_{1}}\left(p_{3}-p_{1}\right)\right] \cos \theta \\
\Delta H & =\Delta G\left(\frac{U_{3}^{2}}{2}+h_{3}\right)
\end{aligned}\right.
$$

where $\eta$ is the mixer efficiency and $\theta$ is the angle at which the flow is injected into the mixer at its internal node (see Fig. 5). Eq. (5) is derived under the hypothesis that the pressure surrounding the injected flow is equal to the pressure at station 1; this can be assured if the mixer module is defined so that the inlet to the module (station 1) is close to the point of injection. 


\section{II.E. Injection}

The injection module takes care of the mixing between the injected fuel and the main air stream. Two models are available; both simply add the fuel flow to the main air flow and do not consider the possibility of any reaction between the fuel and the incoming air at the point of injection. In the first model, the addition of the fuel mass to the flow is accounted for simply by increasing the pressure at the exit to the module over that at the entrance according to

$$
p_{2}=\frac{p_{1}}{1-X_{\text {fuel }}}
$$

where $X_{\text {fuel }}$ is the molar fraction of the fuel. In this case the temperature and velocity are considered constant along the length of the module. Although this assumption is not physically valid it can be useful during validation of the model - as will be explained in IV. The second model is more accurate and makes use of the solver described in II.A.1. The module takes as input the equivalence ratio $\varphi$ together with the fuel temperature and composition and calculates the fluxes needed in Eq. (1) according to

$$
\left\{\begin{aligned}
\Delta G & =-(\varphi \Phi) \frac{W_{f}}{W_{1}}\left(X_{o x}\right)_{1} \rho_{1} U_{1} \\
\Delta I & =0 \\
\Delta H & =\Delta G h_{f}
\end{aligned}\right.
$$

where the principal hypothesis is that the momentum of the injected fuel is negligible compared to that of the main flow.

\section{II.F. Combustion Chamber}

The combustion chamber is probably the most complex part of the engine system to model. In the scramjet case the interaction between several distinct physical phenomena all need to be accounted for simultaneously, rendering the modularization process particularly difficult. In addition, the high temperatures in the combustion chamber and the associated changes in composition of the gas mean that real gas effects cannot be neglected. Moreover, in the case of supersonic combustion, heat exchange and friction are important processes that need to be accounted for. Given the complexity of the physics, very few models for the scramjet combustion process are currently available in the open literature. For these reasons, in the current version of HPPMM, the solver described in II.A.1 is employed once the composition of the gas at the end of the combustion chamber has been calculated assuming complete combustion. ${ }^{4}$

If needed, the heat flux exchanged through the chamber walls and the momentum loss due to friction can be added to the model as discussed in II.A.1. The momentum loss due to friction

$$
\begin{aligned}
\Delta I & =\bar{\tau} \frac{A_{W}}{A_{1}} \\
\text { where } \quad \bar{\tau} & =\frac{\tau_{1}+\tau_{2}}{2} \\
\text { and } \quad \tau_{i} & =\frac{\rho_{i} U_{i}^{2}}{2} C_{f}
\end{aligned}
$$

is calculated after assuming a constant friction coefficient $C_{f}$ along the length of the module. Since the variation of $\tau$ along the combustion chamber is not linear, Eq. (8) is only approximate. It does however yield a good compromise between accuracy and computational effort. Finally, the heat exchange with the walls

$$
\begin{aligned}
\Delta H & =\frac{\bar{q}}{\dot{m}} \frac{A_{W}}{A_{1}} \\
\text { where } \quad \bar{q} & =\frac{q_{1}+q_{2}}{2} \\
\text { and } \quad q_{i} & =\rho_{i} U_{i}\left(h_{i}-h_{W}\right) S t
\end{aligned}
$$

is calculated using a similar procedure as per the friction, where $S t$ is the Stanton number of the flow. 


\section{II.G. Nozzle}

At present, a simple model based on isentropic expansion (see II.A) is used, assuming that the flow through the nozzle can be safely modelled as frozen. ${ }^{4}$ The dissipation of the flow through the nozzle can also be modelled simply by expressing it in terms of a constant stagnation pressure drop.

\section{II.H. System}

The system module takes care of the integration, interconnection and communication between the various sub-modules. Moreover it manages the interface with the rest of the vehicle system, so that this in turn can treat the propulsion system as a self-contained 'black box'. The system module solves sequentially for the states associated with the various modules contained within it, respecting a predefined order of solution of the various modules. In Fig. 6 a typical example of a propulsion system is represented, where the arrows indicate the flow of information through the calculation. Usually the calculation proceeds from upstream to downstream, but when choking occurs within the system then information has to be propagated in the opposite direction to let the upstream flow path adapt to its presence. In Fig. 6 for instance, whenever choking is detected in the mixer module then the intake model has to adapt by spilling out part of the incoming flow, while if choking is detected in the combustion chamber then the injection process has to adapt to reduce the amount of fuel being injected into the engine.

In general it is possible to assign to each module a choking feedback routine that acts to modify the upstream flow in order that the conditions for choking within the system are met precisely. Indeed, whenever choking is detected in one of the engine modules, then an iterative procedure is begun in order to converge the flow properties within all upstream modules on those conditions which allow the exact conditions for choking to be established within the affected module.

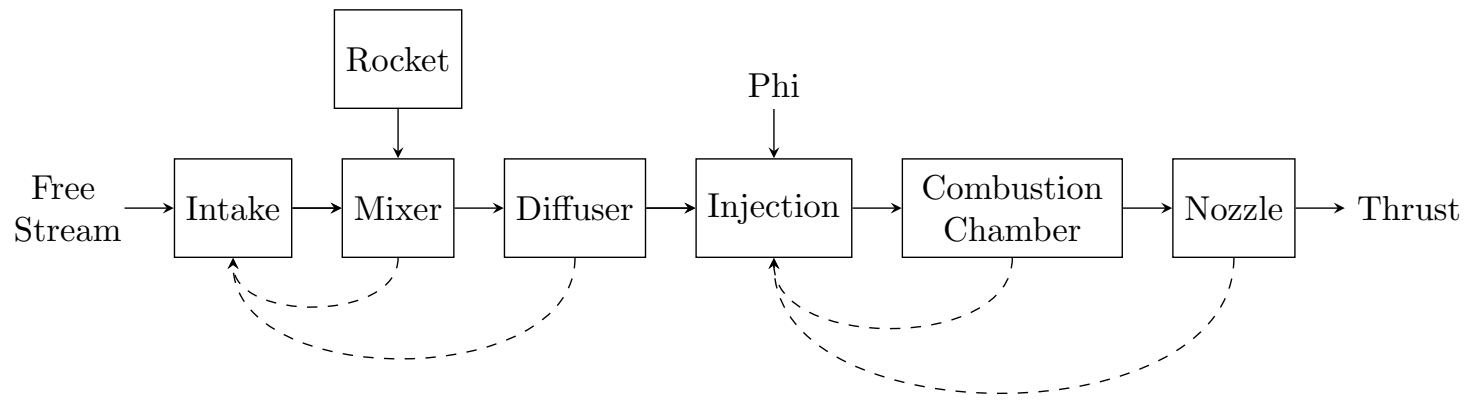

Figure 6. Information flow through the propulsion system model.

Once the flow path is thus fully determined, the global thrust of the engine

$$
T=\dot{m}_{2} U_{2}-\dot{m}_{1} U_{1}+\left(p_{2}-p_{1}\right) A_{2}-D
$$

can be calculated by performing a momentum balance across a convenient control volume. In Eq. (10), subscript 1 indicates the engine inlet, 2 the engine outlet and D is defined in Eq. (3). At present the effect of the flow induced by the engine on the vehicle's external aerodynamics is not considered; this will be added in a future development of the model as a contribution to the overall aerodynamic drag on the vehicle.

\section{Computational Fluid Dynamic (CFD) model}

In addition to its validation against experimental data and other CFD analyses available in the literature, a dedicated CFD campaign has been performed in order to correlate the HPPMM software against more physically complete representations of scramjet behaviour. Of particular importance has been the development of a dedicated CFD model to augment and expand upon the data-sets that are currently available for the model's correlation and verification. The aim has been to create a robust and reliable CFD model that can be used easily to verify the model's performance under particular conditions that might not be fully described in the literature. In addition, as they continue to be developed, the propulsion modules implemented in HPPMM will continually need to be validated and correlated, and the best approach would appear to be to 
compare the predictions of the model to the results of dedicated CFD analyses at various pertinent operating conditions to achieve this objective. To this end, the open source CFD software OpenFOAM@ has been extended and developed to contain a number of algorithms that are appropriate to modelling high-speed propulsion systems. Recently the rhoCentralFoam solver of Greenshields ${ }^{8}$ has been upgraded to yield a new solver called rhoFoam. In this solver, a flux scheme based on the full Riemann solver, using the Godunov approach for the first order approximation and the Weighted Average States (WAS) approach ${ }^{9}$ for the second order approximation to the flow states, has been implemented in order to deal better with the gas dynamic complexities that are inherent to hypersonic flow. The gas model is ideal but with variable specific heat depending on temperature. The specific heat law is given by a polynomial function. ${ }^{10} \mathrm{In}$ all cases described below the flow is considered to be fully turbulent, and the turbulence is modelled using the SST $k-\omega$ model with standard wall functions.

\section{III.A. CFD validation}

In order to verify its predictions, our CFD approach has been used to model the scramjet testbed that was studied by Lorrain ${ }^{11}$ during the development of the SCRAMSPACE I propulsion system. In present context, this test case is particularly helpful given its geometric simplicity and the existence of published analyses of both frozen and reacting flow through the system. Because of this it was possible to verify our CFD model step by step, gradually increasing its complexity. As in Lorrain's work, ${ }^{11}$ our CFD analysis was performed first in a fuel-off condition (see Table 2) in order to gather data for use in the subsequent fuel-on analysis. The intake inlet conditions are listed in Table 1. The results of this preparatory run are illustrated in the pressure contour plot shown in Fig. 7. The fuel-on CFD calculation is performed only for that part of the duct downstream of the intake (see Fig. 8). For equivalence with Lorrain's analysis, the pressure, density and velocity fields produced during the fuel-off computation were interpolated onto the combustion chamber inlet and then applied as boundary conditions for the fuel-on case. Moreover, the pressure downstream of the intake was increased compared to that of the fuel-off case, using Eq. (6) and assuming the flow to be evenly mixed at the outlet of the intake, ${ }^{11}$ in order to take into account the increased mass flow due to the fuel that was injected into the intake of Lorrain's system.

\begin{tabular}{ccl} 
& & Intake Inlet \\
\hline$U$ & {$[\mathrm{~m} / \mathrm{s}]$} & 2830 \\
$p$ & {$[\mathrm{~Pa}]$} & 4100 \\
$T$ & {$[\mathrm{~K}]$} & 370
\end{tabular}

Table 1. Flow conditions at the inlet of the intake. ${ }^{11}$

\begin{tabular}{cll} 
Species & Fuel-Off & Fuel-On \\
\hline $\mathrm{H}_{2}$ & 0.0 & 0.250 \\
$\mathrm{O}_{2}$ & 0.209 & 0.157 \\
$\mathrm{~N}_{2}$ & 0.791 & 0.593
\end{tabular}

Table 2. Mixture composition in terms of molar fraction.

A contour plot of the pressure in the fuel-on case, assuming frozen conditions, is depicted in Fig. 8. As can be seen by comparing the data presented in Fig. 9, the results from our CFD model compare very well with Lorrain's calculations under the assumption of frozen flow through the entire system. Slight differences between the two calculations are indeed evident, however, in the region of the nozzle. At this stage, only this frozen computation has been completed; the calculation of the fully-combusting case will be presented in a later work.

\section{III.B. Supercharged Ejector ScramJet (SERJ) mixer analysis}

The CFD model described in III was used to analyze the performance of a typical ejector engine, in order to provide data to validate and correlate the HPPMM model. The system that was analysed was Marquardt's SERJ engine ${ }^{3,12,13}$ dating from the early 1960s. The engine was of Rocket-Based Combined Cycle (RBCC) configuration and was envisaged to form the propulsion system for a proposed Two Stage to Orbit (TSTO) 


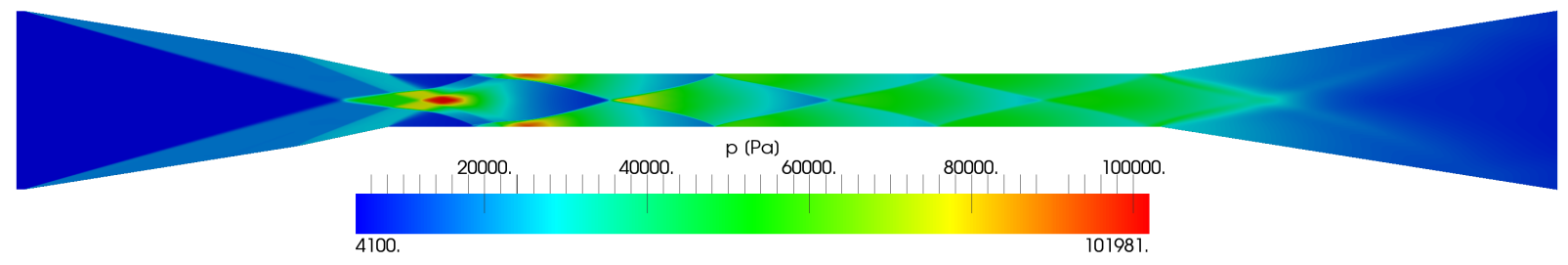

Figure 7. RhoFoam CFD results for Lorrain's geometry in fuel-off conditions, showing pressure contours [Pa] within the engine duct.

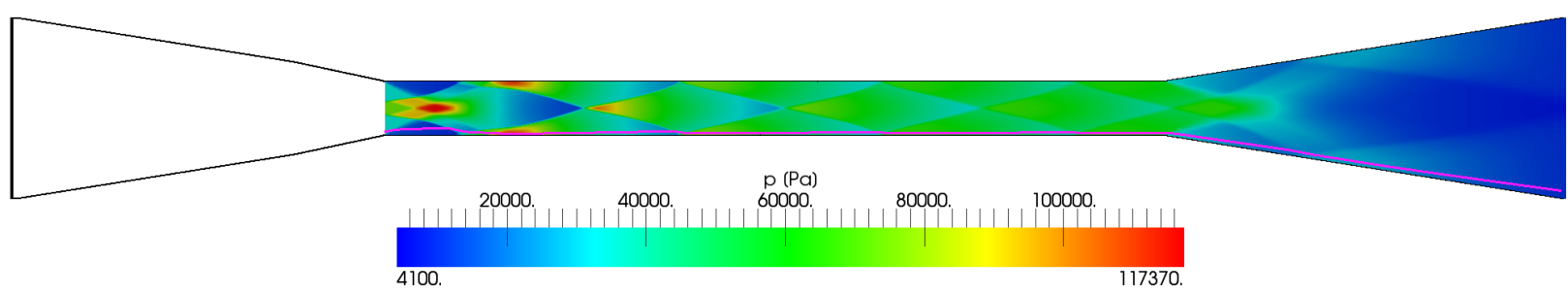

Figure 8. RhoFoam CFD results for Lorrain's geometry in fuel-on conditions, with frozen chemistry, showing pressure contours $[\mathrm{Pa}]$ within the engine duct.

vehicle. The engine was developed specifically to work efficiently over a wide range of flight Mach numbers by changing its configuration to allow operation in several different modes: ${ }^{13}$

- Supercharged ejector mode

- Fan ramjet mode

- Pure ramjet mode

- Pure rocket mode

- Fan operation mode

The engine is provided with an annular $\mathrm{H} 2 / \mathrm{O} 2$ rocket and a post-combustor, and has a retractable fan to enhance its performance at low speed (see Fig. 10).

The CFD analysis presented here evaluates only the performance of the ejector-mixer section of the Marquardt engine: many aspects of this system's rather complex configuration are thus neglected. As illustrated in Fig. 11, the CFD calculation domain starts downstream of the fan and extends up to the nozzle exit. The flow through the ejector-mixer is considered to be axially symmetric throughout. The flow medium is assumed to be air with constant composition, thus combustion and the mixing of gas species is not considered in the results presented here. The post-combustor is also considered not to be active in this case. On the other hand, the primary rocket is considered to be active, but under the simplification that its working fluid, and hence the composition of the plume that it generates, is air. It is important to note that this assumption does not compromise the final purpose of the analysis, since mixing within the system is affected primarily by the momentum of the flow in the plume and only secondarily by its composition. 


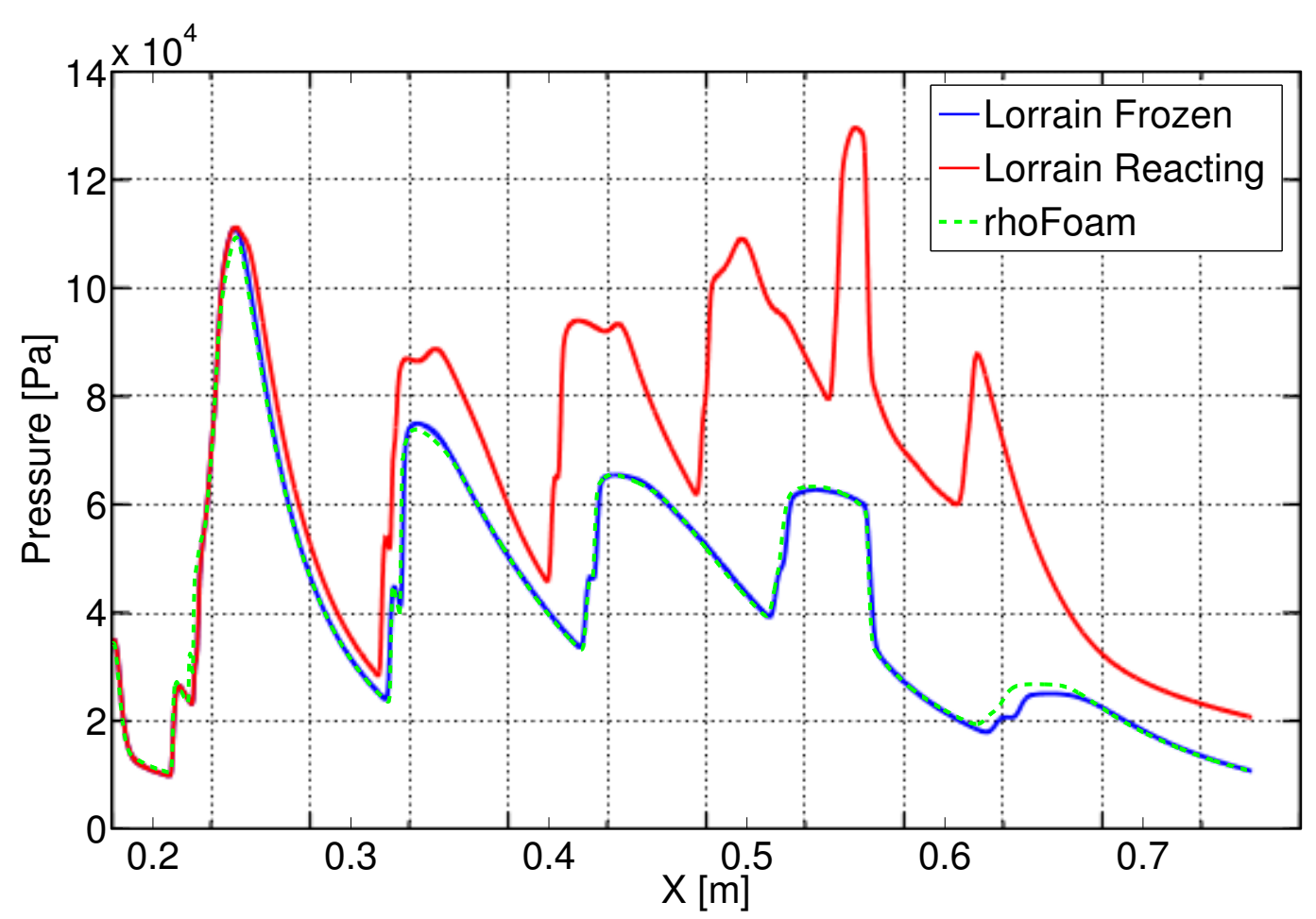

Figure 9. Comparison between rhoFoam predictions and Lorrain's ${ }^{11}$ calculations. The pressure profile is calculated along a pathline close to the combustion chamber wall (see Fig. 8).

The boundary conditions for the calculation are selected to reproduce the working conditions experienced by the engine at sea level and at Mach $0.75 .{ }^{13}$ The total mass flow rate and the stagnation temperature are assigned at the inlet (see Table 3), while the other quantities are calculated by the solver. The pressure, temperature and velocity at the rocket outlet are directly specified, once calculated from the known values of the total mass flow rate through the rocket nozzle and the rocket chamber pressure and temperature (see Table 3). Since the post-combustor is not included in this model, the nozzle throat area is reduced in order to let the engine work at the same mass flow rate and pressure of the actual configuration. Hence the nozzle plug is positioned accordingly (see Fig. 11). All walls are all assumed to be adiabatic, and as such the results presented here do not account for the influence of any thermal losses upon the performance of the engine.

\begin{tabular}{ccl} 
& & Rocket Outlet \\
\hline$U$ & {$[\mathrm{~m} / \mathrm{s}]$} & 2623 \\
$p$ & {$[\mathrm{~Pa}]$} & 40094 \\
$T$ & {$[\mathrm{~K}]$} & 1062.9 \\
$\dot{m}$ & {$[\mathrm{~kg} / \mathrm{s}]$} & 177.8 \\
$p_{0}$ & {$[\mathrm{~Pa}]$} & $1.034 \cdot 10^{7}$ \\
$T_{0}$ & {$[\mathrm{~K}]$} & 3675 \\
\hline & & Inlet \\
\hline$\dot{m}$ & {$[\mathrm{~kg} / \mathrm{s}]$} & 743.4 \\
$T_{0}$ & {$[\mathrm{~K}]$} & 620.2
\end{tabular}

Table 3. Marquardt SERJ boundary conditions ${ }^{13}$

The pressure and velocity contours inside the engine duct, calculated using RhoFoam, are shown in Figs. 12 and 13. Fig. 13 shows in addition the flow path lines within the engine. The flow within the duct is essentially driven by the shear interaction between the rocket plume and the remainder of the fluid in the system, and this entrainment mechanism is responsible for many of the observed features of the flow. 


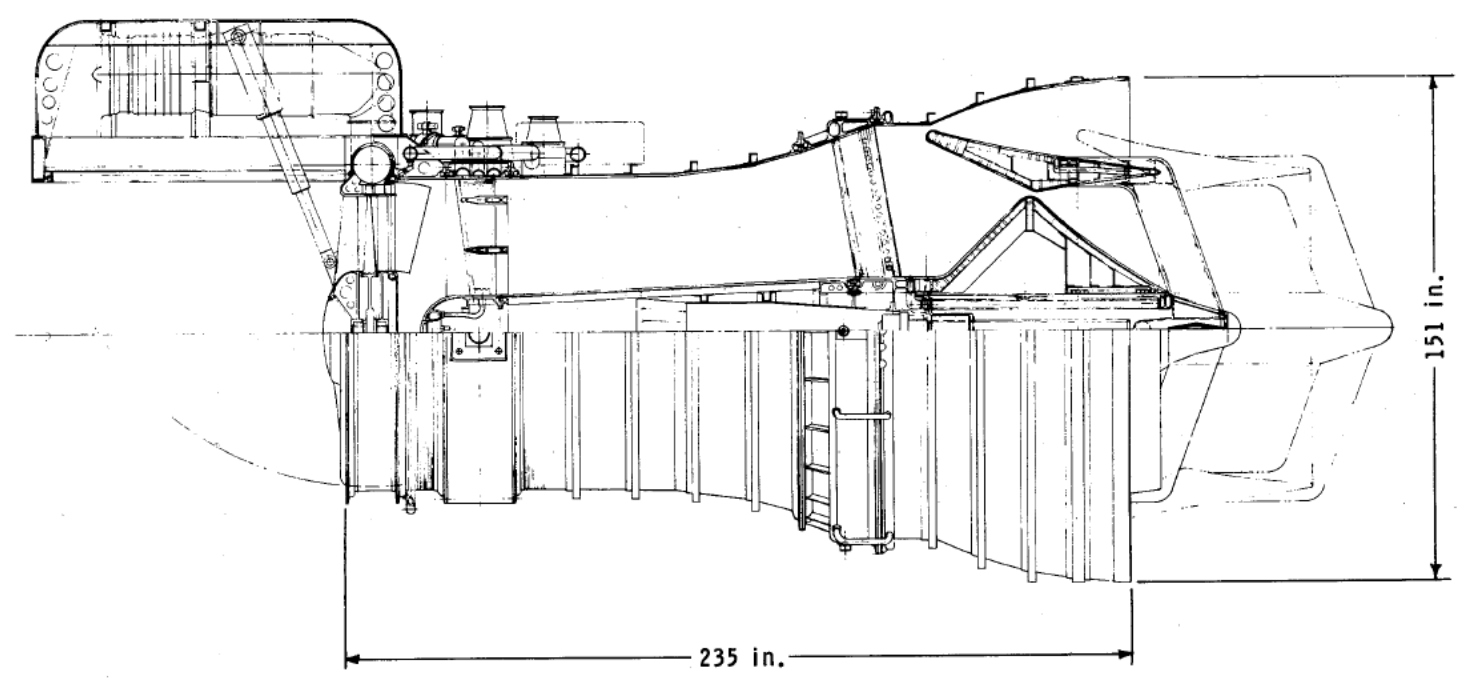

Figure 10. Overall view of the Marquardt SERJ engine. ${ }^{3}$

Indeed, a predominant feature of the flow within the duct is the large separation bubble near the end of the mixer: the presence of this phenomenon leads to a very low mixer efficiency (see IV.C). It appears that the adverse pressure gradient that is produced by the addition of the flow through the rocket ejector is primarily responsible for the separation of the inner wall boundary layer and thus the production of the separation bubble. This interpretation is supported by the observation that, because of the distance of the inner wall from the rocket plume, the kinetic energy of the flow there is very low - indeed, separation does not occur on the upper wall of the duct that is much closer to the plume. It is interesting to speculate on how this behaviour could be avoided (or indeed if this flow feature was known to the original developers of the engine given the state of their analysis tools some fifty years ago). Ostensibly a different design of the ejector with enhanced momentum transfer to the central part of the engine might have been sufficient to eliminate the separation bubble - indeed, there is evidence to suggest that a twin-nozzle ejector system was also considered by Marquardt during the evolution of their design (see Fig.10). In present context, however, the occurrence of these interesting secondary flows serves admirably to exercise and validate the capabilities of HPPMM indeed, the presence of these complex adverse conditions yield an opportunity to demonstrate the flexibility and the robustness of the software (see IV.C).

An interesting contour plot, perhaps allowing the origins of separation phenomena within the ducts of engine configurations such as this to be understood a little better, is shown in Fig. 14. In this figure, the temperature contours within the duct, together with the flow path lines of the solution obtained using the second-order RhoFoam scheme as described above, are compared against the solution that is obtained using first order discretization (i.e. using solely the Godunov flux scheme ${ }^{9}$ throughout the computational domain). It appears that, because of the higher diffusivity of the first order method compared to the full WAS flux scheme used in the RhoFoam algorithm, more kinetic energy is transferred to the inner wall boundary layer from the rocket jet and the separation is thus not predicted to take place. In Figs. 15 and 16 the pressure and Mach number for both the first- and the second-order solutions are plotted along the engine axis (after averaging across each axial station of the duct). These figures show conclusively how the presence of the separation bubble acts to reduce the performance of the ejector (see IV.C).

As a final confirmation of the performance of our CFD approach, in particular regarding the insensitivity of its predictions to the discretization of the computational domain, results obtained using two different mesh densities (Mesh A with 21,232 cells, and Mesh B, with 84,928 cells obtained by subdividing the cells of Mesh A) are also compared in Figs. 15 and 16. Although small differences between the two calculations are present, these differences are much smaller than the differences between the solutions with and without the separation bubble. The level of mesh insensitivity demonstrated in these figures is entirely adequate in the present context, particularly given the magnitude of some of the other likely sources of error within our approximation to the engine geometry and our treatment of the composition of its internal flow. 


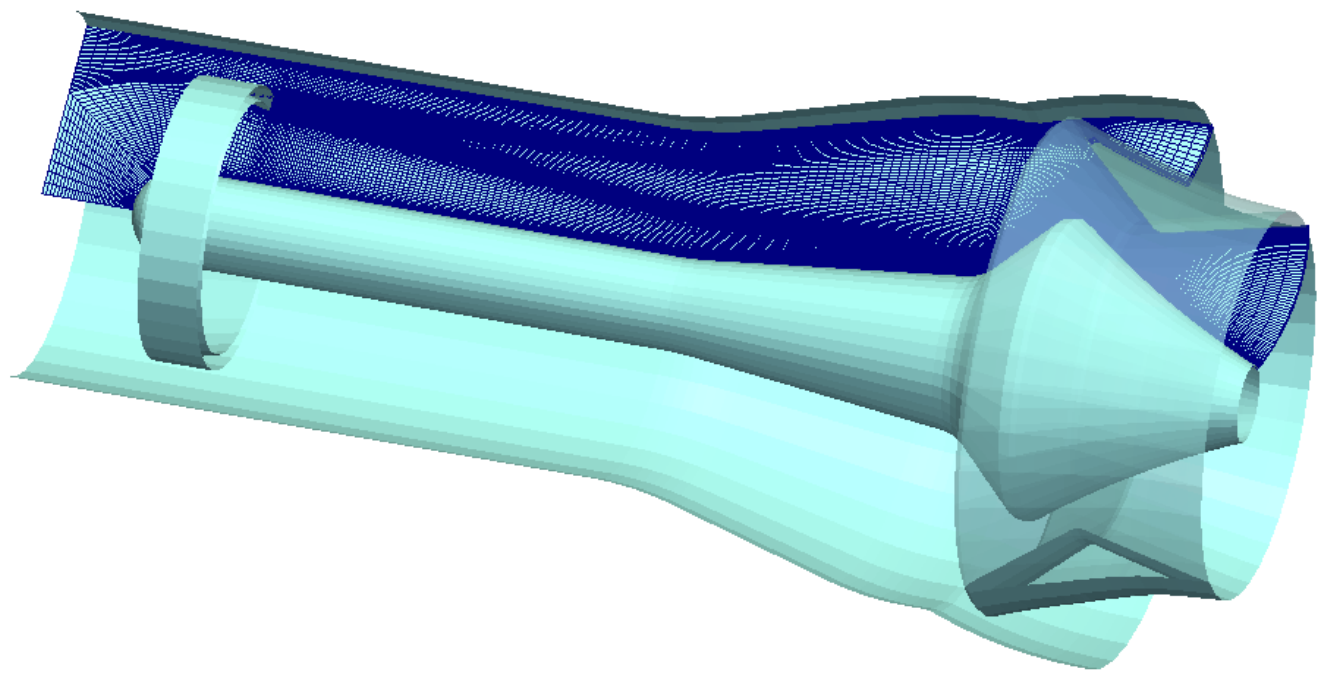

Figure 11. CFD model discretization of the Marquardt SERJ mixer.

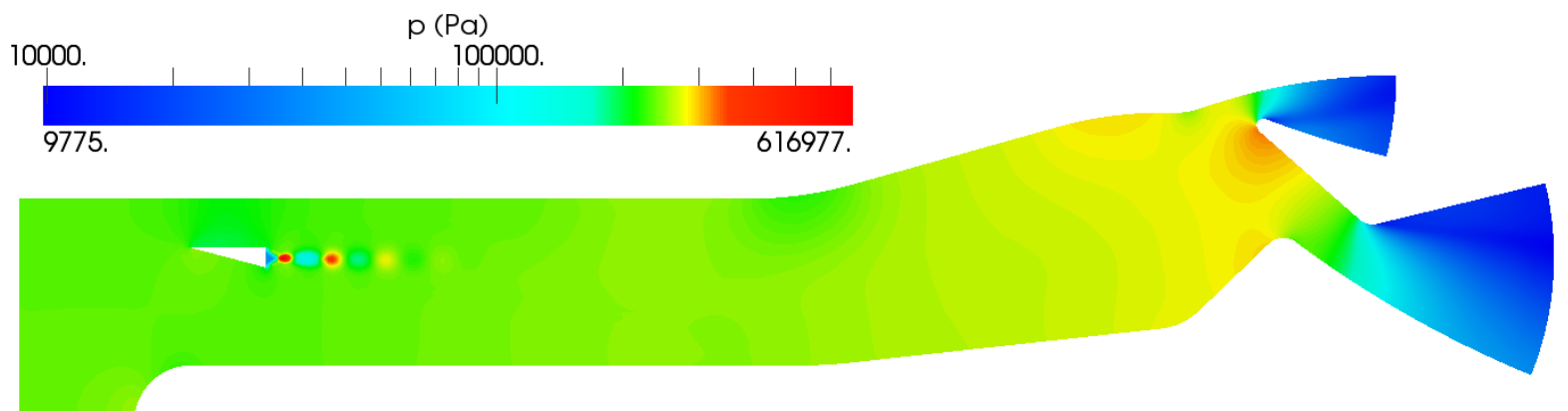

Figure 12. CFD results for the Marquardt SERJ mixer: static pressure contours. 


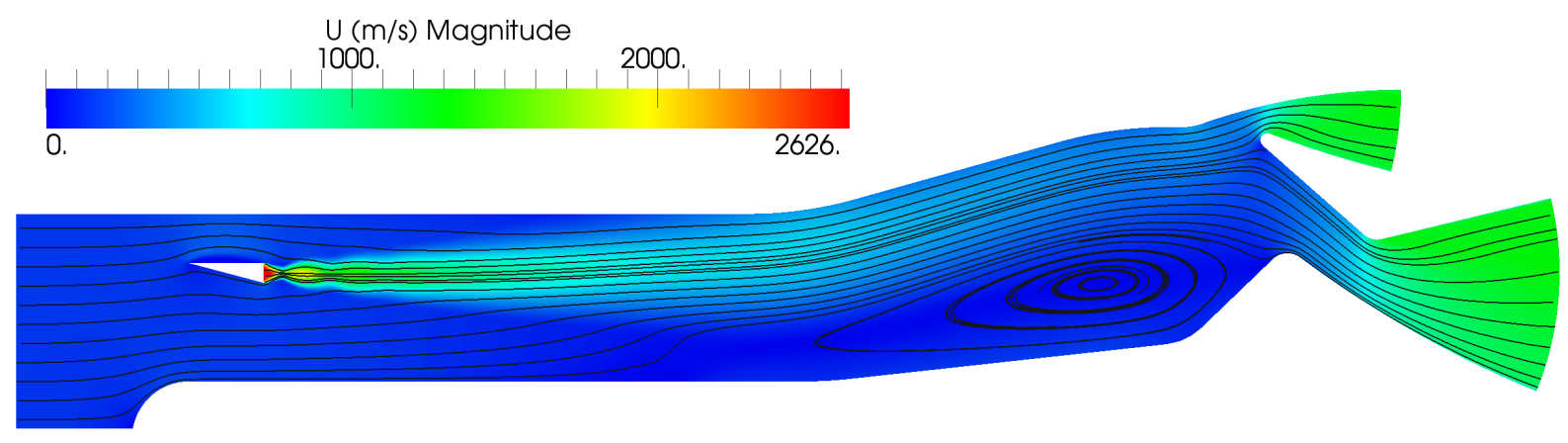

Figure 13. CFD results for the Marquardt SERJ mixer: velocity contours and flow path lines.

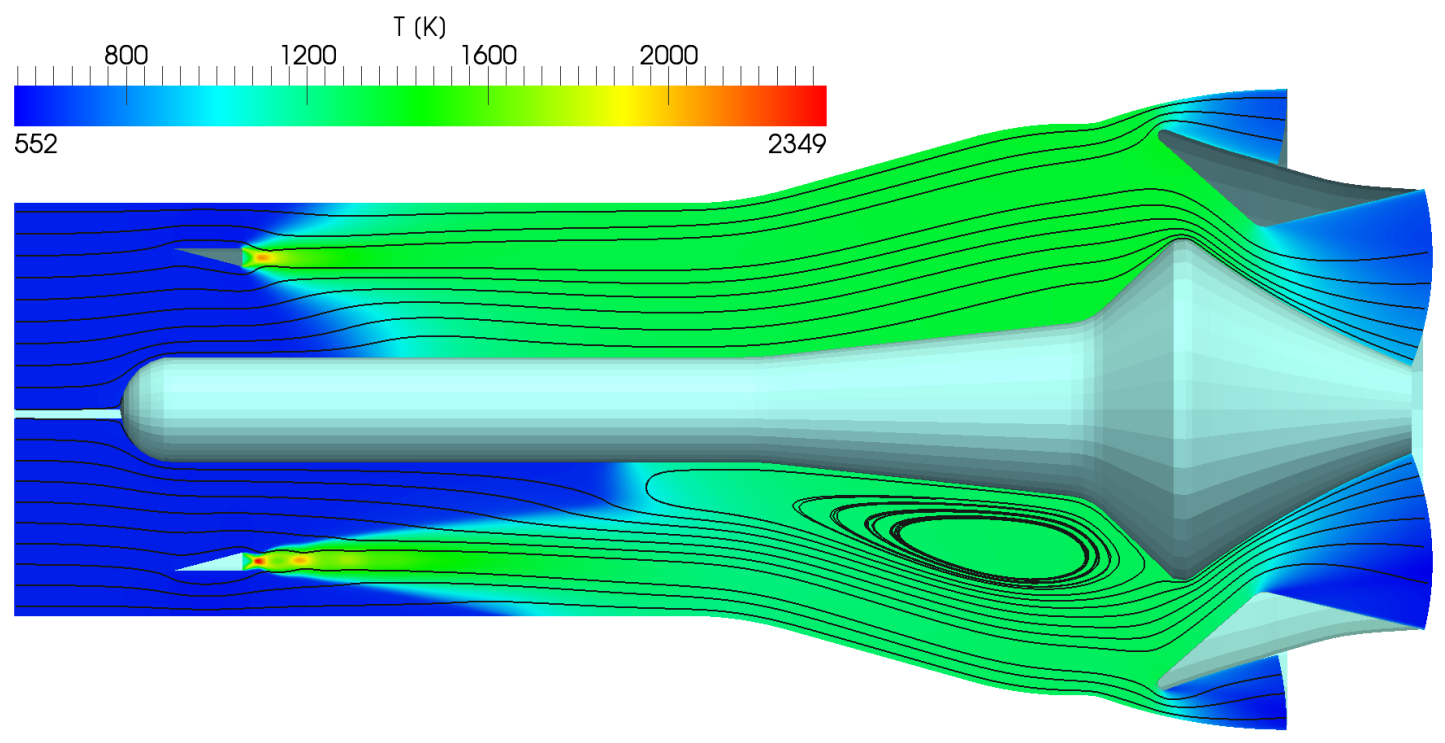

Figure 14. CFD results for the Marquardt SERJ mixer: static temperature contours and flow path lines. Top half: First order Godunov flux scheme. Bottom half: Second order WAS flux scheme. 


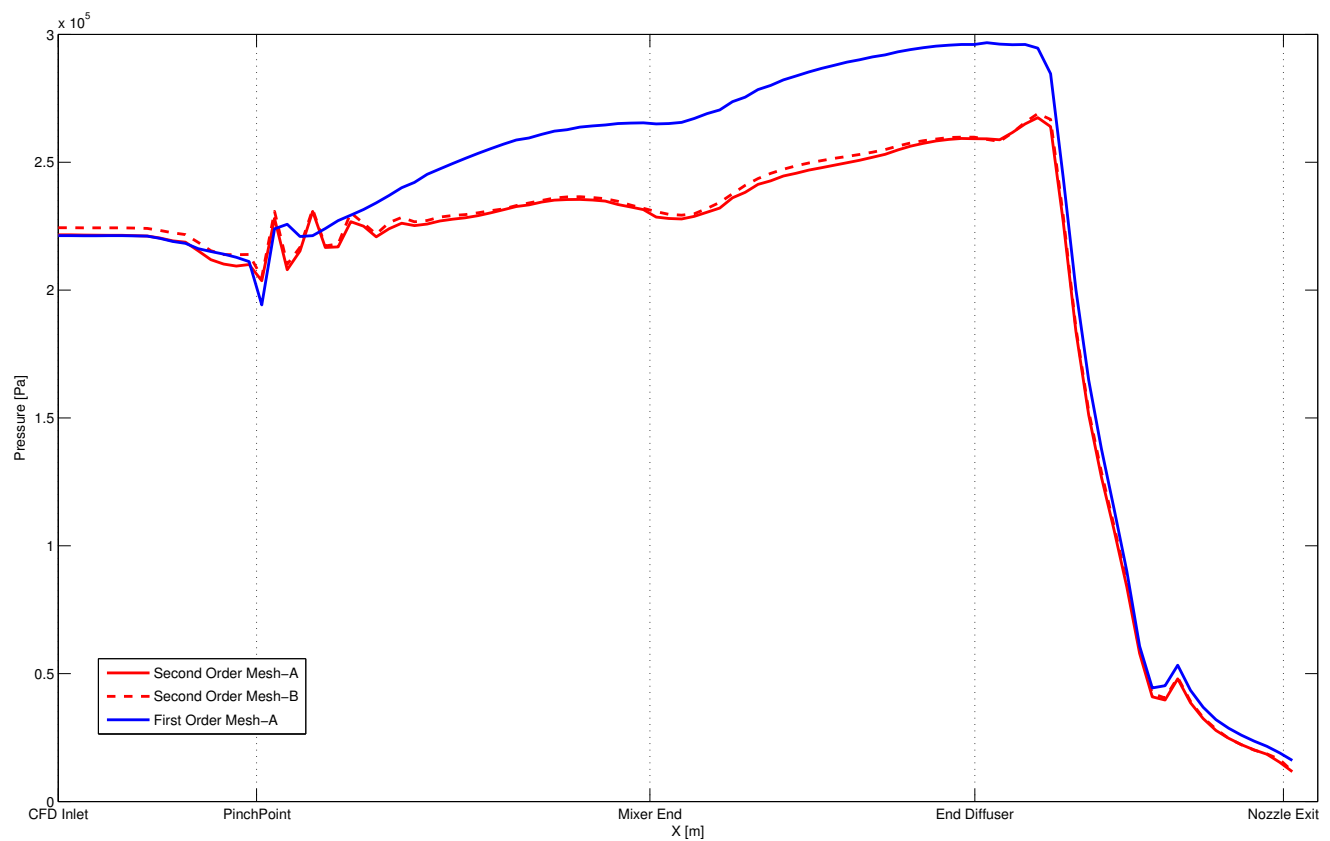

Figure 15. CFD results for the Marquardt SERJ mixer: section-averaged static pressure along the engine axis.

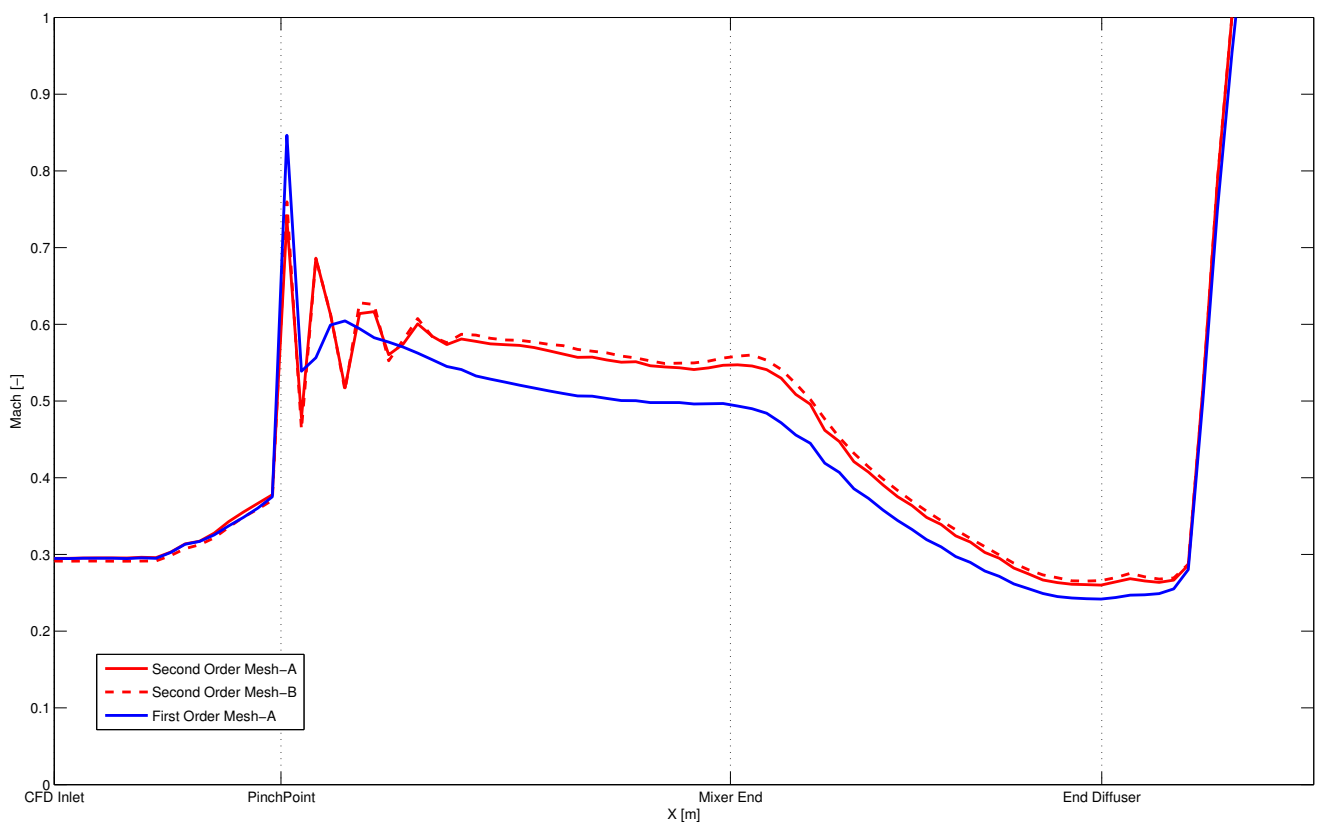

Figure 16. CFD results for the Marquardt SERJ mixer: section-averaged Mach number along the engine axis. 


\section{HPPMM Validation and comparisons}

In order to provide a preliminary validation of its predictions, the HPPMM software in its present state has been used to model three test cases for which data are available from either experimental sources, other CFD analyses or the predictions of other simplified models.

\section{IV.A. SCRAMSPACE I test case}

The first test case to be described is the scramjet testbed that was studied by Lorrain ${ }^{11}$ during the development of the SCRAMSPACE I propulsion system. Lorrain's testbed is a channel with constant width, a two-slope intake, a constant area combustion chamber and a single-slope nozzle. ${ }^{11}$ The testbed was operated in a shock tunnel using air and hydrogen as fuel. The fuel was injected into the intake in order to facilitate pre-mixing prior to combustion, and to take advantage of the concept of 'radical farming' for ignition of the fuel-air mixture. ${ }^{11,14,15}$ To account for these features, the injection point in the HPPMM model of the testbed (see Fig. 17) has been placed downstream of the intake, and it is assumed that the pre-mixing of the fuel and air is completed entirely within the intake itself. The radical farming process is modelled by adding a pre-combustion module before the combustion chamber itself. The pre-combustion process is modelled using a simple Fanno flow module (see II.A) with $C_{f}=0.0015$. The pre-combustion module takes into account the fact that, in a scramjet that employs the radical farming strategy, combustion does not occur at the inlet of the combustion chamber, but only downstream of the first shock interaction within the duct. ${ }^{14,11,15}$ The combustion module assumes full combustion with wall friction (see II.F) assuming again that $C_{f}=0.0015$.

A comparison between HPPMM predictions and the CFD calculations performed by Lorrain ${ }^{11}$ is illustrated in Fig. 18. The HPPMM results can be seen to be in good agreement with those produced by the CFD analysis, once it is realised that the CFD results are for the pressure profile along a flow path line that passes close to the engine wall, while the HPPMM data, coming as it does from a quasi one-dimensional model, represents better the averaged flow quantities at each axial station along the engine duct. Indeed, a better comparison between the two models might result if the pressure calculated in Lorrain's CFD ${ }^{11}$ were to be averaged across several engine stations, but the intrinsic difference in fidelity between the two types of model, particularly in terms of their ability to reperesent the detailed features of the flow, should be borne firmly in mind when evaluating the results presented here.

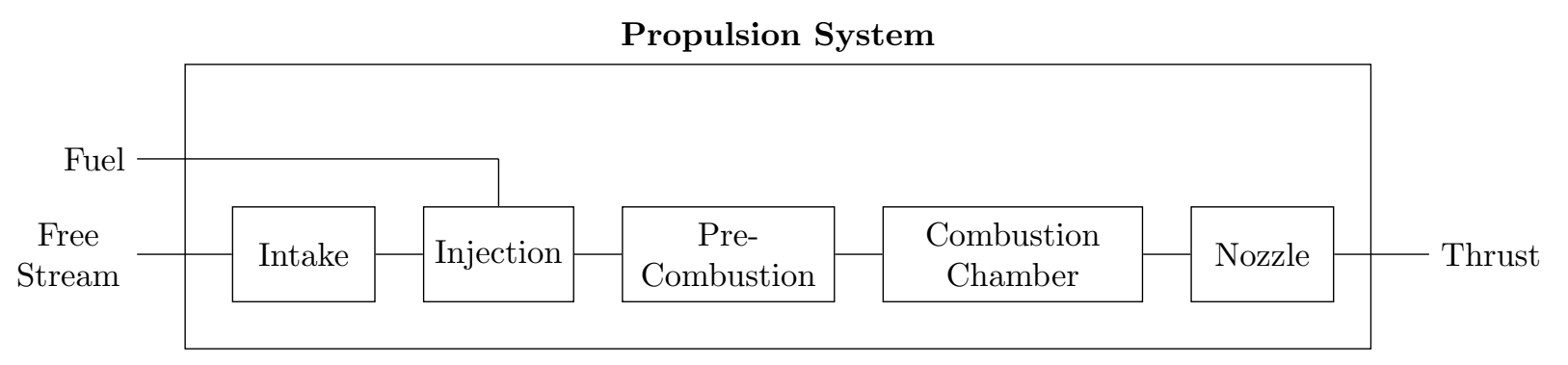

Figure 17. HPPMM model structure used to reconstruct Lorrain's scramjet testbed.

\section{IV.B. Hyperion test case}

A further validation case is presented in order to compare the predictions of HPPMM against those of a similar software called the Simulated Combined-Cycle Rocket Engine Analysis Module (SCCREAM). ${ }^{4,7,16}$ This software was used to evaluate the performance of the ejector ramjet engine that was proposed for Hyperion, a launch vehicle conceived by the Aerospace Systems Design Laboratory at Georgia Tech. ${ }^{4}$ An HPPMM model representing the ejector mode of the Hyperion propulsion system (and reproducing the work of $\mathrm{Olds}^{4}$ ) is depicted in Fig. 19.

The intake is modelled as fully adaptable, in other words with the possibility that both throat and inlet have a section area that can be varied with the operational condition of the engine. The flow is assumed always to be choked at the throat of the intake, that is reduced in size if choking occurs in one of the the downstream modules. The inlet section is assumed instead to be adapted to the external field conditions if possible (i.e. if the required inlet area is within the design range). An isentropic diffuser is added after 


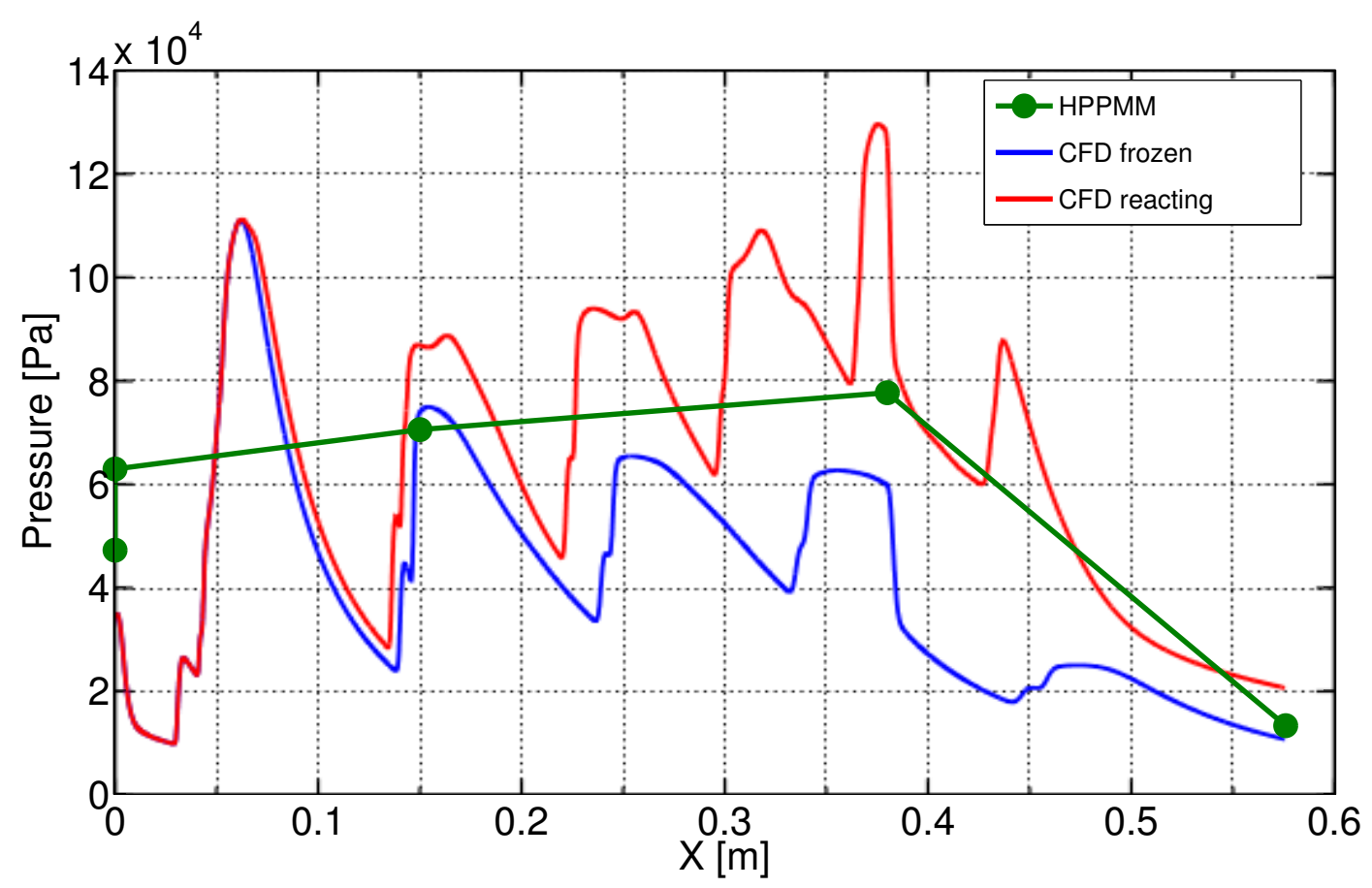

Figure 18. Comparison between the axial pressure profile predicted by HPPMM with combustion, and results from Lorrain's CFD calculations. ${ }^{11}$

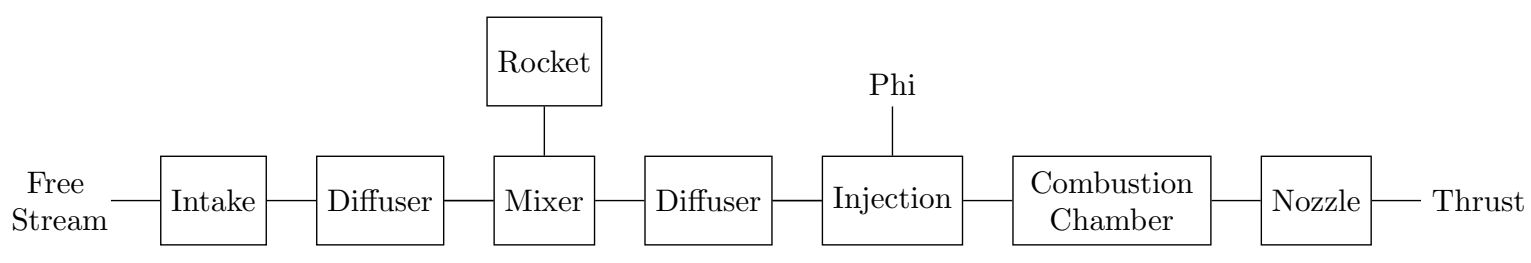

Figure 19. HPPMM model of the Hyperion ejector ramjet engine.

the intake module in order to be able to model a pinch point area (at the end of the intake module) that is smaller than the mixer section area. The injection of the rocket plume is assumed to occur along the axis of the engine (see II.D). The rocket is not modelled in any detail however: its nozzle exit conditions are considered constant and are fed directly into the calculations within the mixer module. After the flow passes through a second isentropic diffuser, injection takes place assuming a constant $\varphi$ followed by combustion (see II.E and II.F). The equivalence ratio $\varphi$ within the injection module can be reduced if choking is detected downstream of the injection point (see II.H). A full description of the distribution of nodal areas along the axis of the engine is given in Table 4, bearing in mind that both the inlet throat and intake as well as the nozzle exit section have variable area. For the purposes of validation, the thrust predicted by the HPPMM model was matched to that predicted ${ }^{4}$ by the SCCREAM model at sea level and Mach 0 by tuning the mixer efficiency to have a value of $67 \%$.

A comparison between HPPMM and SCCREAM predictions ${ }^{4}$ of thrust and specific impulse for the Hyperion engine when operated in ejector mode over a range of altitudes and Mach numbers is shown in Figs. 20 and 21. Despite, or in fact because of, the tuning of the mixer efficiency in the HPPMM model, the thrust predicted by HPPMM matches reasonably well with that predicted by SCCREAM, at least in trend even if nonetheless being consistently somewhat overoptimistic in magnitude. Comparison of the predictions by the two models of the specific impulse of the Hyperion engine leads to similar conclusions, bearing in mind that the predictions of the two models were not tuned to yield a closer match with respect to fuel consumption. The discrepancies between their predictions are thought most likely to be due to the presence of a number of unresolved differences between the rocket primary input data, and its variation with altitude, assumed by the two models. 


\begin{tabular}{cll} 
& \multicolumn{2}{c}{ Area $\left[\mathrm{m}^{2}\right]$} \\
Node name & Min & Max \\
\hline Pre-Intake & 0 & $\infty$ \\
Intake & 0 & 2.5084 \\
Throat & 0 & 0.6271 \\
Pinch Point & 0.7655 & 0.7655 \\
Primary & 1.0452 & 1.0452 \\
Mixer End & 1.0452 & 1.0452 \\
Rocket Outlet & 0.2796 & 0.2796 \\
End Diffuser & 2.0903 & 2.0903 \\
Injection & 2.0903 & 2.0903 \\
End Chamber & 2.0903 & 2.0903 \\
Nozzle & 0 & 8.8258
\end{tabular}

Table 4. Nodal areas within the HPPMM model of the Hyperion engine.

Further insight into the performance of the HPPMM model are given in the plots shown in Fig. 22, 23 and 24, where results for operation of the Hyperion engine at Mach 0 and at Mach 2.5 are contrasted at sea level and at an altitude of about $20 \mathrm{~km}$. The intake is predicted to be fully opened at Mach 0 , although, since the capture area tends to infinity the intake will not have adapted fully to the external flow conditions. On the other hand a much smaller intake area is sufficient at Mach 2.5 to guarantee that the inlet is adapted to the external flow conditions. As shown in Fig. 23, the working pressure of the engine changes dramatically in passing from Mach 0 to Mach 2.5 at sea level. It is worth noting however that the pressure at the end of the mixer is the same for all operating conditions with the exception of Mach 2.5 at sea level; this is because the secondary flow is always much smaller than the primary flow (i.e. the flow in the rocket exhaust plume), with the exception of at this particular flight condition where the dynamic pressure of the free stream is much greater. Finally, Fig. 24 illustrates the conditions under which the flow becomes choked within the engine. In interpreting this diagram it is important to realise that the flow is considered to be choked within the model if the Mach number approaches 0.9 rather than unity - this choice gives stability to the code and accounts for viscous effects that in the real system would reduce the available area for the flow under choked conditions.

\section{IV.C. Marquart SERJ test case}

The performance of ejector type engines seems to be heavily influenced by the efficiency of the ejector (see IV.B). In this section, the results of the CFD analysis described in III.B for the Marquardt SERJ engine are compared to the predictions of the dedicated HPPMM model shown in Fig. 25 in order to investigate this sensitivity. The CFD boundary conditions are fed as direct inputs into the HPPMM model in order to compare the predictions of the two approaches under the same operating conditions. In the HPPMM implementation of the SERJ mixer, the mixer module itself is preceded by an isentropic convergent duct in order to account for the change in cross section area between the section after the fan and the primary section (see III.B). Another isentropic duct downstream of the mixer module simulates the diffuser of the engine, and the flow through the nozzle is also considered to be isentropic. Thus, in the analysis presented here, only the mixer is considered to have an efficiency lower than unity. For consistency with the CFD analysis (see III.B), the post-combustor is not modelled even though the capacity to do so exists within the HPPMM toolbox.

Fig. 26 shows a comparison between the results of our CFD analysis and the predictions of the HPPMM model for the Marquardt SERJ mixer. The CFD data are averaged at each section along the axis of the engine in order to yield results that are directly compatible with the one dimensional nature of the HPPMM predictions. As can be seen, the CFD analysis and the HPPMM predictions are in very close agreement all the way up to the mixer pinch point, while some small discrepancies are present within the mixer itself. In particular, the results of the first order CFD calculation are in very good agreement with the predictions of the HPPMM model when no account is taken of any inefficiency within the duct (i.e. when the HPPMM mixer efficiency is set equal to unity, see II.D), the HPPMM results showing just a slight overestimation of 


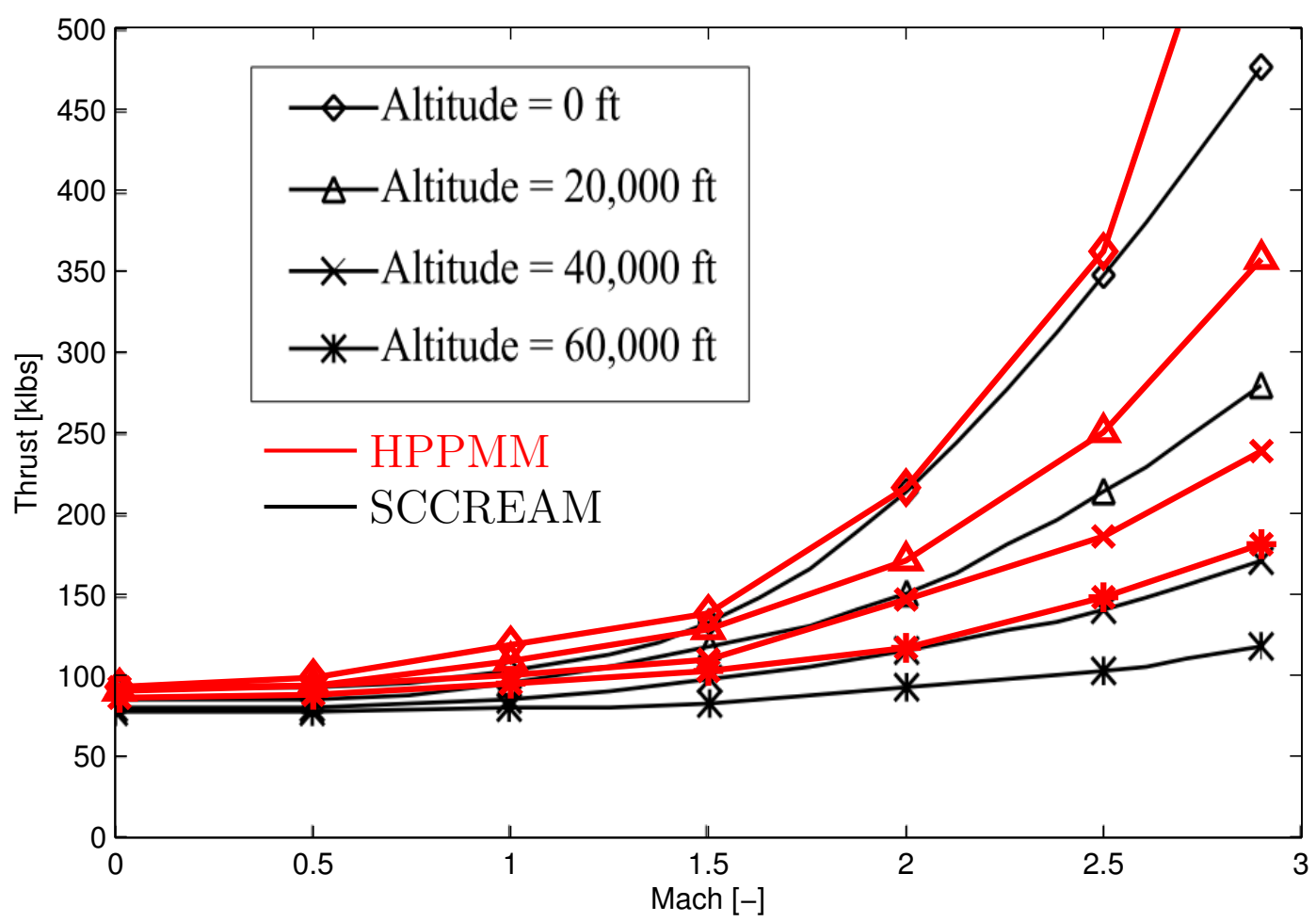

Figure 20. Comparison between HPPMM and SCCREAM predictions of the thrust of the Hyperion engine in ejector mode. $^{4}$

performance due to the unrealistic assumption of a perfect mixer. The second order CFD analysis yields a much lower predicted performance for the engine - mainly, as postulated in III.B due to the strong separation that is predicted to occur within the mixer. Good correlation between the two models can be restored however if the mixer efficiency within the HPPMM model is tuned to have a value of $70 \%$, as shown in Fig. 26. Indeed, by tuning this parameter alone it is possible to obtain very good correlation between the CFD analysis and the reduced-order HPPMM model along the entire length of the flow path within the engine. This lends significant confidence in the robustness of the HPPMM model in being able to capture the performance of complex, hybrid propulsion systems even in the presence of extreme and non-optimal conditions, such as this large-scale separation, within the flow path of the engine. 


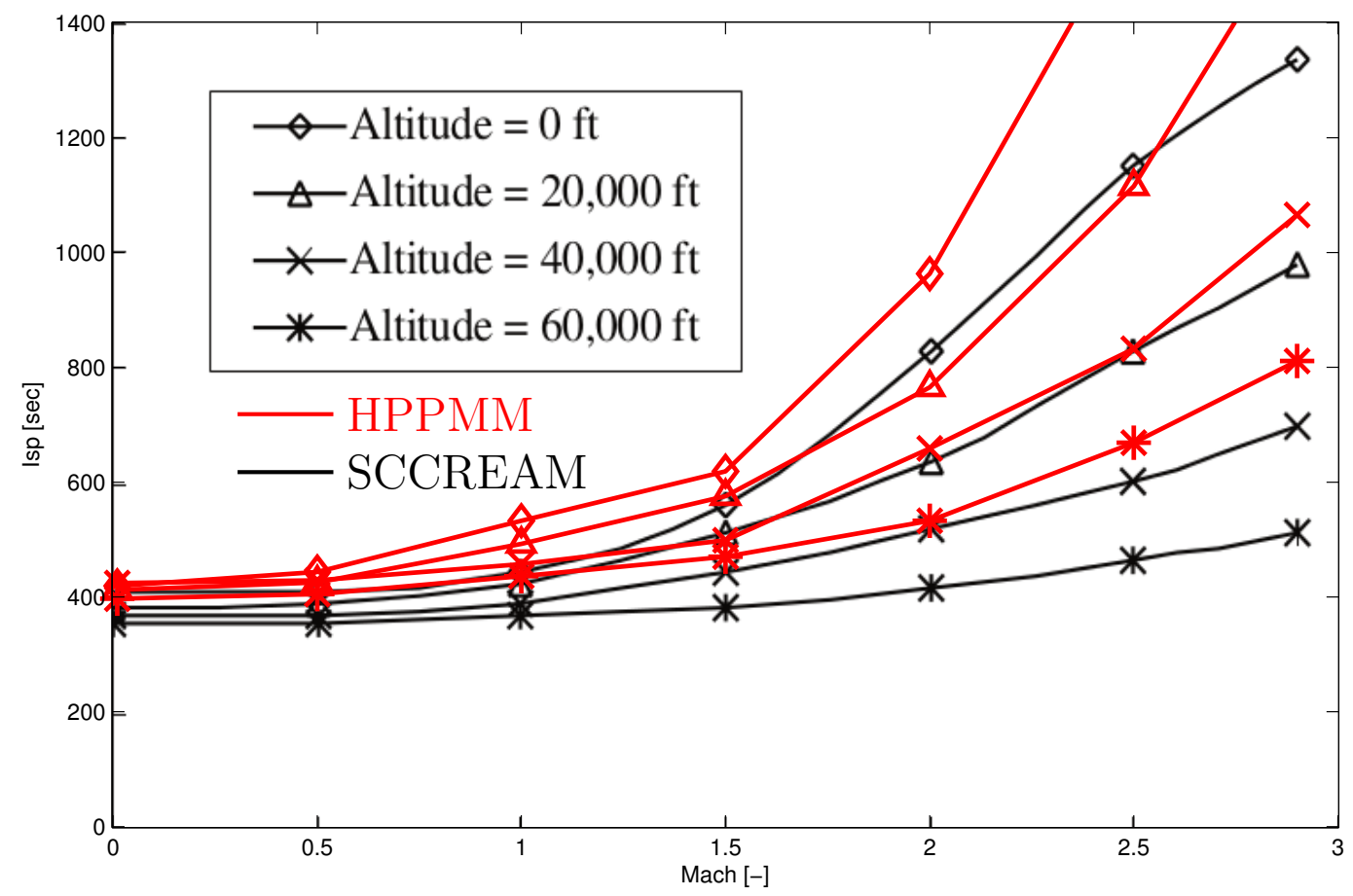

Figure 21. Comparison between HPPMM and SCCREAM predictions of the specific impulse of the Hyperion engine in ejector mode. ${ }^{4}$

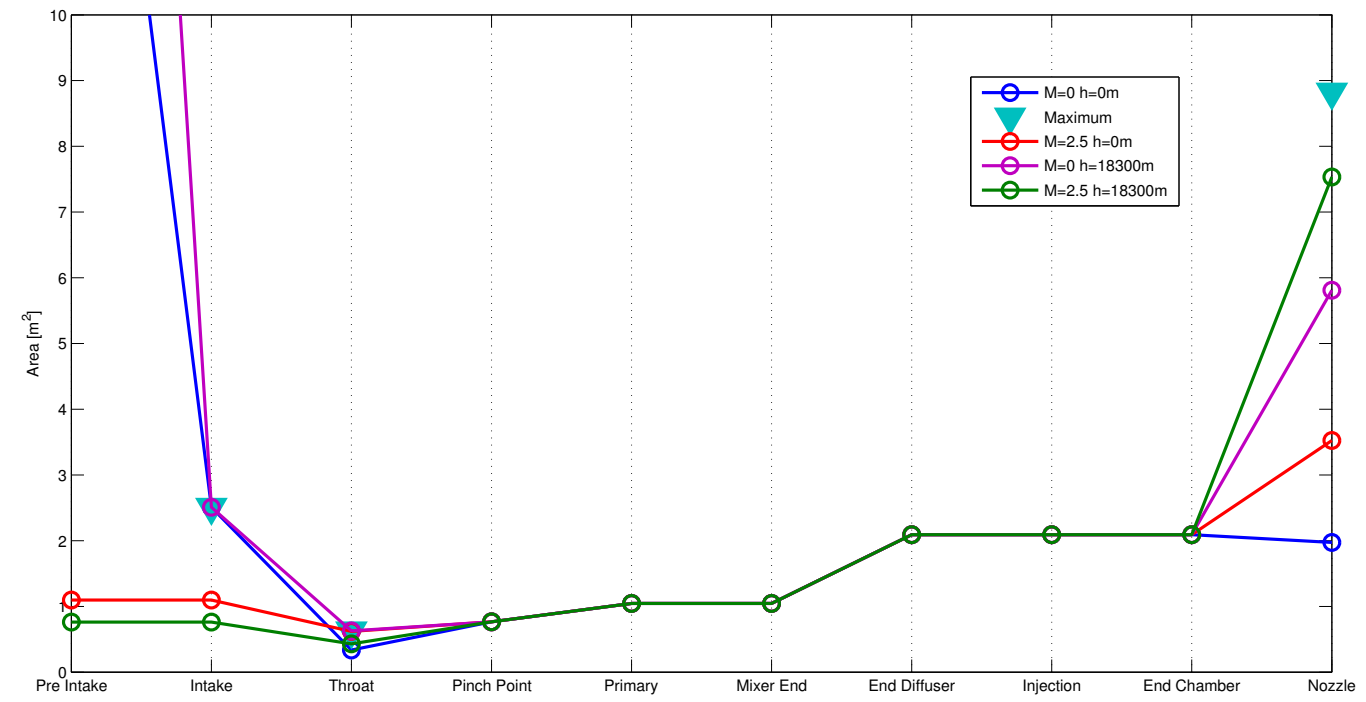

Figure 22. HPPMM predictions of the variation of section area along the length of the Hyperion engine when operated in ejector mode. 


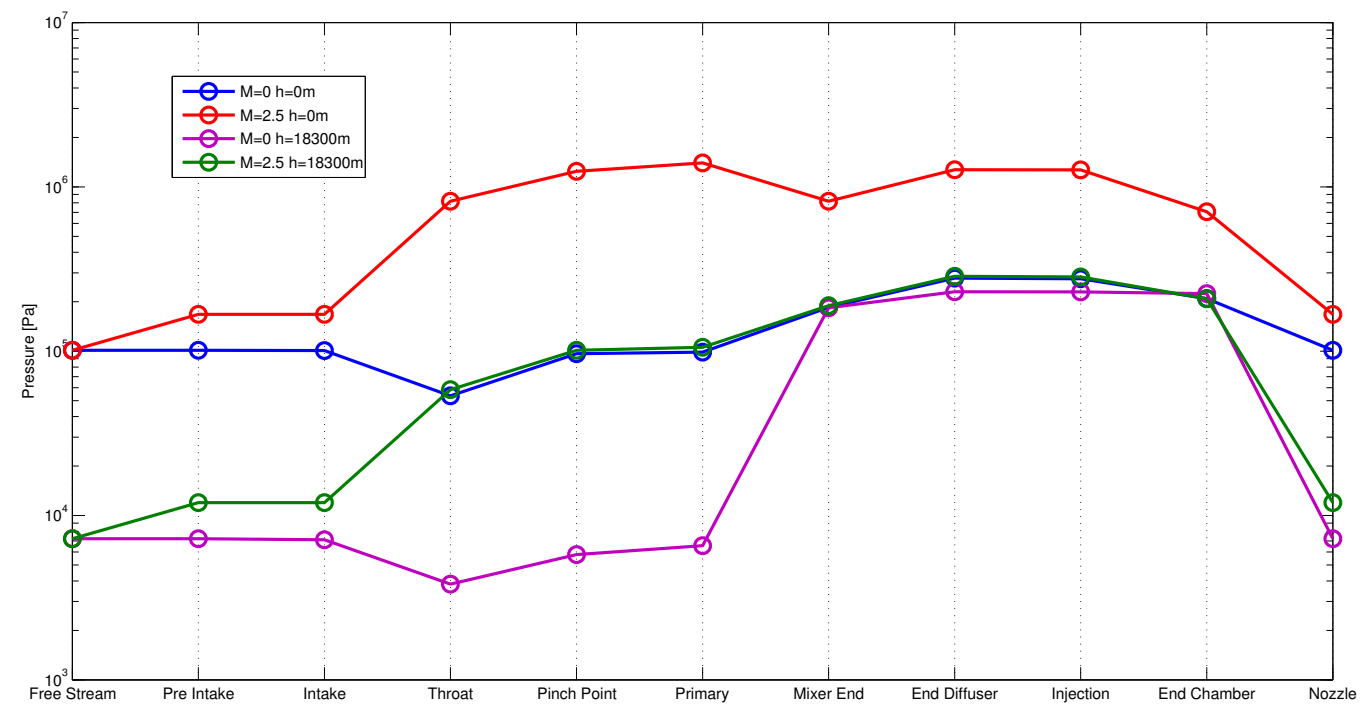

Figure 23. HPPMM predictions of the variation of static pressure along the length of the Hyperion engine when operated in ejector mode.

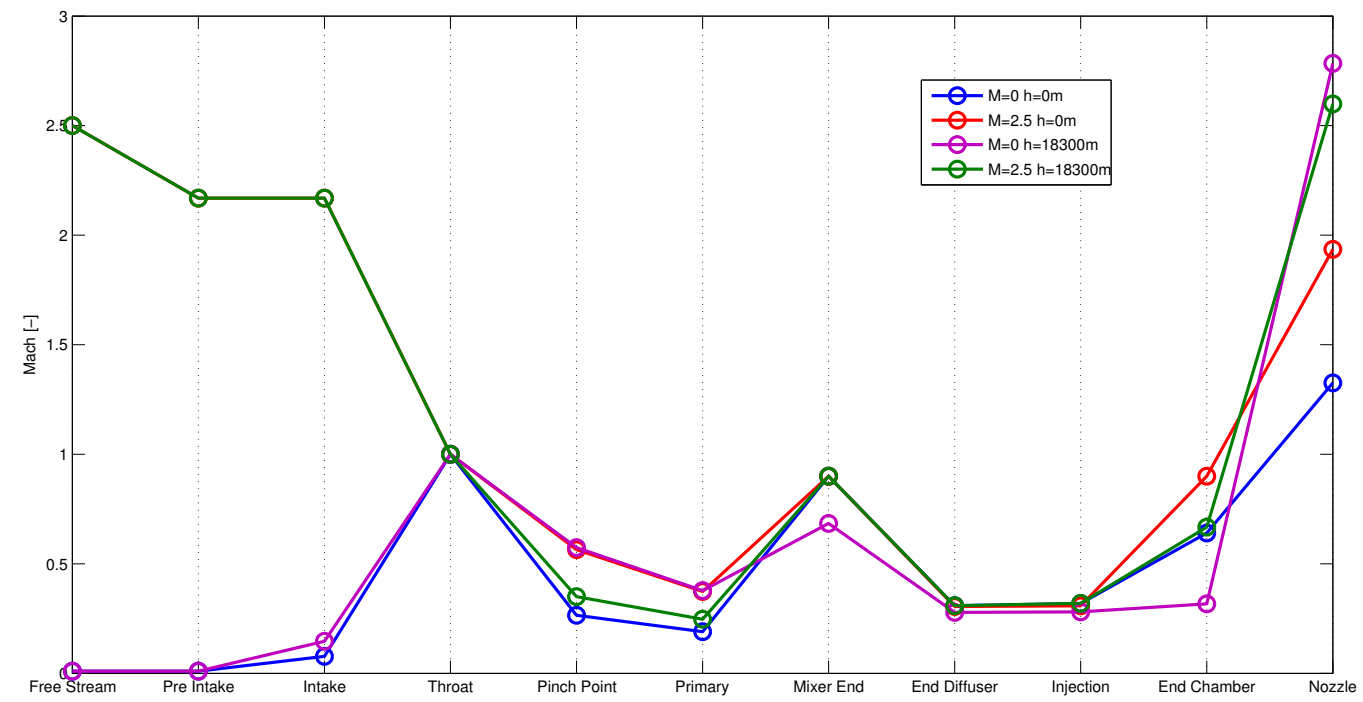

Figure 24. HPPMM predictions of the variation of Mach number along the length of the Hyperion engine when operated in ejector mode.

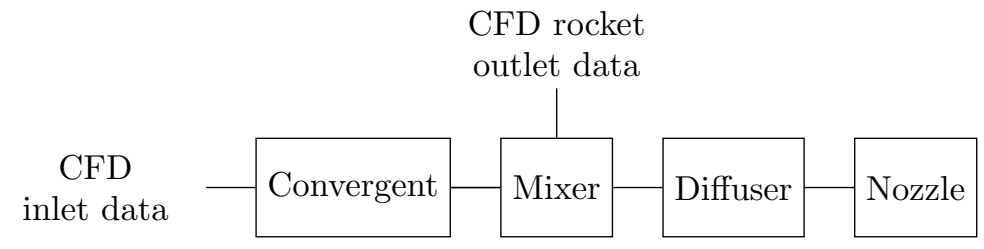

Figure 25. System diagram for the Marquardt SERJ mixer as replicated in HPPMM. 


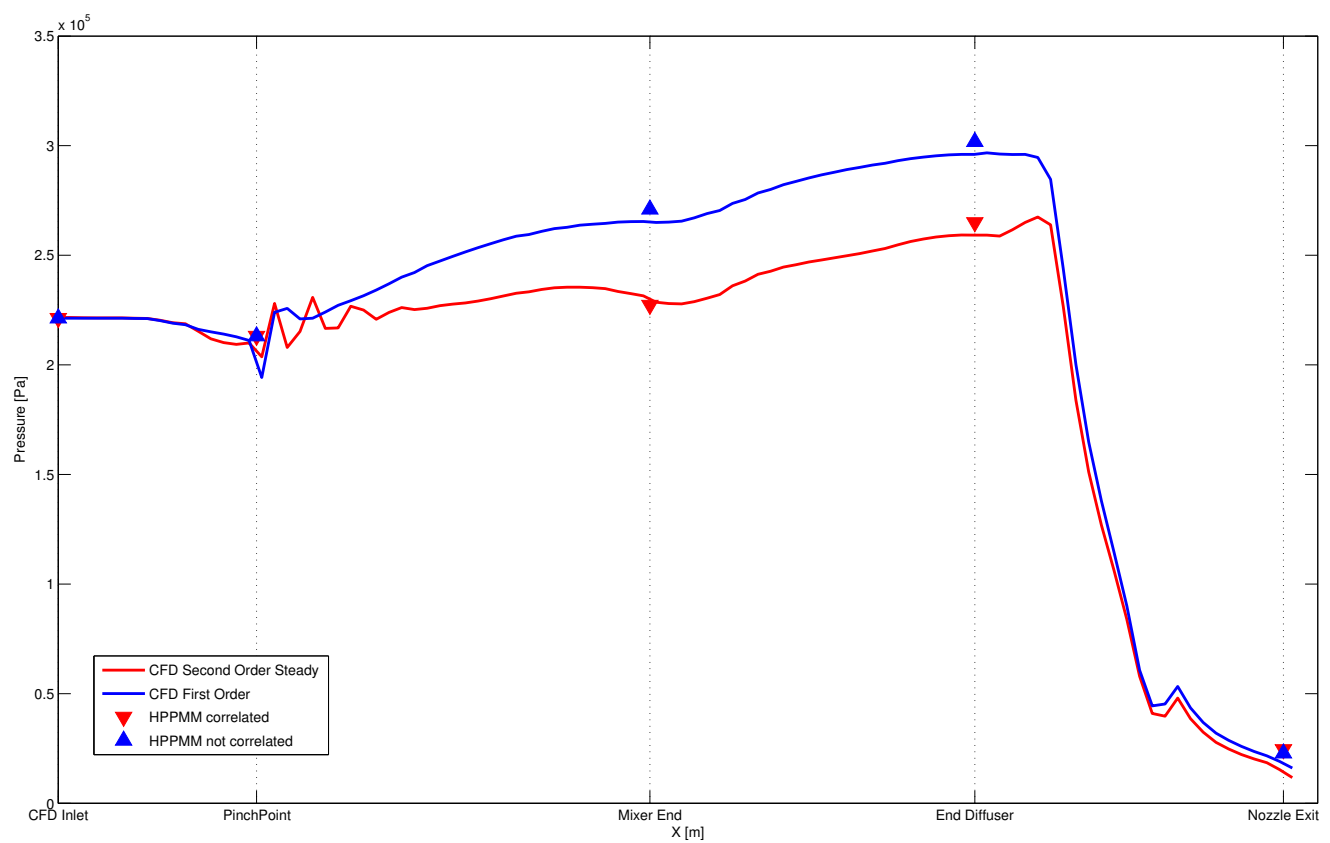

Figure 26. SERJ Marquardt Mixer, comparison CFD vs HPPMM: section averaged pressure plot along engine axis.

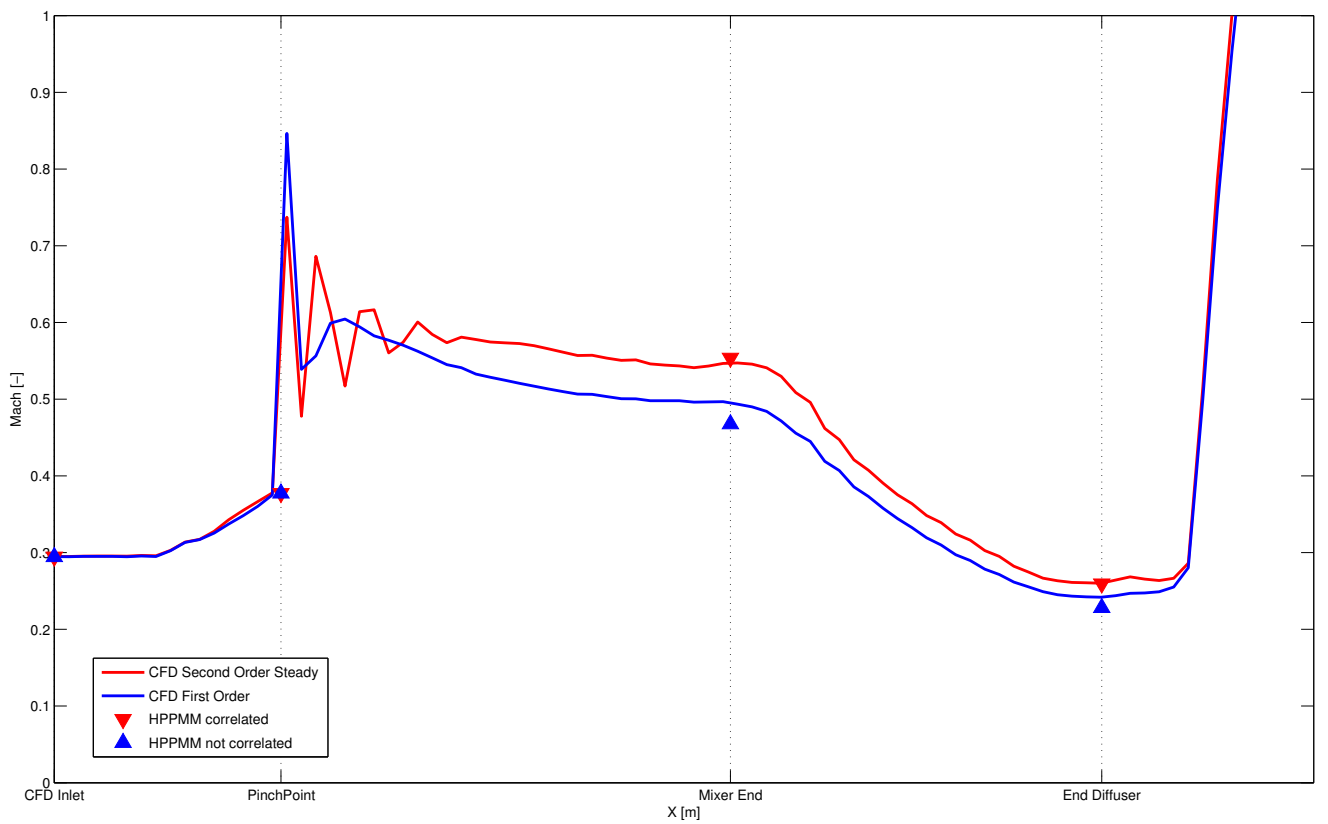

Figure 27. SERJ Marquardt Mixer, comparison CFD vs HPPMM: section averaged Mach number plot along engine axis. 


\section{Conclusion}

A simplified propulsion system modeling tool, called the Hybrid Propulsion Parametric-Modular Model, or HPPMM, has been developed and tested against published scramjet CFD data with promising results. In parallel, an independent CFD model has been developed and validated against the same data, presently under frozen flow conditions, but with the aim of extending the analysis to be able to model the effects on performance of the fully-reacting real gas flow through the propulsion system.

This CFD code has been used to generate data in order to further validate HPPMM - in this work the particular a case of an ejector engine has been analyzed and HPPMM has been successfully correlated against CFD predictions of the internal flow within the system. Furthermore HPPMM has been compared against the predictions of a similar code called SCCREAM with satisfactory results, demonstrating its applicability to engines that have complex internal configurations.

Our aim is to use the simplified model in conjunction with our new CFD tool to extend our understanding of the performance of hypersonic propulsion systems, particularly those with hybrid configuration, and to encapsulate this understanding into an engineering analysis tool that can be applied to the Multi-disciplinary Design Optimization of the next generation of Re-usable Launch Vehicles.

\section{References}

${ }^{1}$ Curran, E. T., "Scramjet Engines: The First Forty Years," Journal of Propulsion and Power, Vol. 17, 2001, pp. 1138-1148.

${ }^{2}$ Tran, K., One Dimensional Analysis Program for Scramjet and Ramjet Flowpaths, Master's thesis, University Libraries, Virginia Polytechnic Institute and State University, Blacksburg, VA, 2010.

${ }^{3}$ Escher, W. J. and Flornes, B. J., "A Study of Composite Propulsion Systems for Advanced Launch Vehicle Applications," Vol. 1, The Marquardt Corporation, Van Nuys, CA, 1966.

${ }^{4}$ Olds, J. and Bradford, J., "SCCREAM (Simulated Combined-Cycle Rocket Engine Analysis Module): A Conceptual RBCC Engine Design Tool," 33rd AIAA/ASME/SAE/ASEE Joint Propulsion Conference 83 Exhibit, Seattle, WA, 6 - 9 July 1997.

${ }^{5}$ Yamaguchi, H., Engineering Fluid Mechanics, Springer, 2008.

${ }^{6}$ Carlomagno, G. M., Elementi di Gasdinamica, Liguori, 2009.

${ }^{7}$ Bradford, J. and Olds, J., "Improvements and Enhancements to SCCREAM, a Conceptual RBCC Engine Analysis Tool," 34 th AIAA/ASME/SAE/ASEE Joint Propulsion Conference 83 Exhibit, Cleveland, OH, 13-15 July 1998.

${ }^{8}$ Greenshields, C., Weller, H., Gasparini, L., and Reese, J., "Implementation of Semi-Discrete, Non-Staggered Central Schemes in a Colocated, Polyhedral, Finite Volume Framework, for High-Speed Viscous Flows," International Journal for Numerical Methods in Fluids, Vol. 63, No. 1, 2010, pp. 1-21.

${ }^{9}$ Toro, E. F., Riemann Solvers and Numerical Methods for Fluid Dynamics: A Practical Introduction, Springer, 2009.

${ }^{10}$ McBride, B. J., Gordon, S., and Reno, M. A., "Coefficients for Calculating Thermodynamic and Transport Properties of Individual Species," NASA TM-4513, National Aeronautics and Space Administration, Washington D.C., 1993.

${ }^{11}$ Lorrain, P., Brieschenk, S., Capra, B., and Boyce, R., "A Detailed Investigation of Nominally 2-D Radical-Farming Scramjet Combustion," 18th AIAA/3AF International Space Planes and Hypersonic Systems and Technologies Conference, Tours, France, 24 - 28 September 2012.

${ }^{12}$ Escher, W. J. and Flornes, B. J., "A Study of Composite Propulsion Systems for Advanced Launch Vehicle Applications," Vol. 3, The Marquardt Corporation, Van Nuys, CA, 1966.

${ }^{13}$ Escher, W. J. and Flornes, B. J., "A Study of Composite Propulsion Systems for Advanced Launch Vehicle Applications," Vol. 7, The Marquardt Corporation, Van Nuys, CA, 1966.

${ }^{14}$ Gardner, A. D., Paull, A., and McIntyre, T. J., "Upstream Porthole Injection in a 2-D Scramjet Model," Shock Waves, Vol. 11, No. 5, 2002, pp. 369-375.

${ }^{15}$ McGuire, J., Ignition Enhancement for Scramjet Combustion, Ph.D. thesis, University of New South Wales, Australian Defence Force Academy, School of Aerospace and Mechanical Engineering, 2007.

${ }^{16}$ Bradford, J. and Olds, J., "SCCREAM v. 5: a Web-Based Airbreathing Propulsion Analysis Tool," 35th AIAA/ASME/SAE/ASEE Joint Propulsion Conference and Exhibit, Georgia Institute of Technology, Los Angeles, CA, 20-24 June 1999. 